\title{
DISCRETE-TIME SEMI-MARKOV RANDOM EVOLUTIONS AND THEIR APPLICATIONS
}

\author{
NIKOLAOS LIMNIOS, ${ }^{*}$ Université de Technologie de Compiègne \\ ANATOLIY SWISHCHUK, ${ }^{* *}$ University of Calgary
}

\begin{abstract}
In this paper we introduce discrete-time semi-Markov random evolutions (DTSMREs) and study asymptotic properties, namely, averaging, diffusion approximation, and diffusion approximation with equilibrium by the martingale weak convergence method. The controlled DTSMREs are introduced and Hamilton-Jacobi-Bellman equations are derived for them. The applications here concern the additive functionals (AFs), geometric Markov renewal chains (GMRCs), and dynamical systems (DSs) in discrete time. The rates of convergence in the limit theorems for DTSMREs and AFs, GMRCs, and DSs are also presented.
\end{abstract}

Keywords: Semi-Markov chain; random evolution; averaging; diffusion approximation; diffusion approximation with equilibrium; rates of convergence; additive functional; geometric Markov renewal process; dynamical system; controlled random evolution; controlled additive functional and geometric Markov renewal chain; optimal control; Hamilton-Jacobi-Bellman equation

2010 Mathematics Subject Classification: Primary 60K15; 60B10; 90C40; 60F17

Secondary $60 \mathrm{~K} 37$

\section{Introduction}

Random evolution is a very powerful technique to study stochastic evolutionary systems; see, e.g. [13], [14], [18], [19], [28], and [47]. Discrete-time random evolutions, induced by discrete-time Markov chains, are introduced in [17] (see also [38] and [49]) and discrete-time semi-Markov random evolutions are introduced in [23]. Swishchuk and $\mathrm{Wu}$ [47] and Koroliuk and Limnios [18] introduced discrete-time random evolutions induced by the embedded Markov chain of continuous-time semi-Markov processes. This is equivalent to discrete-time Markov random evolution stopped in random time (see also Remark 2.1 below).

Discrete-time semi-Markov chains (SMCs) have only recently been used in applications, specifically, in DNA analysis, image and speech processing, reliability, etc.; see [5] and the references therein. These applications have stimulated a research effort in this area. However, while the literature in discrete-time Markov chains theory and applications is huge, there is only a very small number of works on SMCs and most of these are in hidden semi-Markov models for estimation.

In this paper we consider the general case of discrete-time semi-Markov random evolution with value in a Banach space, say $\mathbb{B}$, and we present their limit theory in series (functional) scheme. In particular, we obtain weak convergence theorems in Skorokhod space $D_{\mathbb{B}}[0, \infty)$ for

Received 27 September 2011; revision received 25 May 2012.

* Postal address: Laboratoire de Mathématiques Appliquées, Université de Technologie de Compiègne, BP 20529, 60205 Compiègne Cedex, France. Email address: nikolaos.limnios@utc.fr

** Postal address: Department of Mathematics and Statistics, University of Calgary, 2500 University Drive NW, Calgary, Alberta T2N 1N4, Canada. 
càdlàg stochastic processes; see, e.g. [15] and [21]. We consider both averaging and diffusion approximation limit theorems, with the latter being of two kinds: equilibrium about a point and equilibrium about a function (or stochastic process) obtained by the averaging limit. We present some results on convergence rates of the above limit theorems, and some additional results on controlled discrete-time semi-Markov random evolutions. Finally, we give some applications of the above results for particular well-known stochastic systems: additive functionals, geometric Markov renewal chains, and discrete-time dynamical systems switched (driven) by SMCs. It is worth noting that dynamical systems have been considered in continuous- and discrete-time cases by Skorokhod [37] and Skorokhod et al. [38], respectively, but, in both cases, with Markov switching and by direct methods. Integral functionals were considered via direct methods by several authors in the semi-Markov case; see, e.g. [35]. Applications of geometric Markov renewal processes in finance have been considered in [43], [44], and [45]. Optimal stopping of geometric Markov renewal processes and pricing of European and American options for underlying assets modeled by geometric Markov renewal processes have been studied in [46].

Results presented here are new and concern discrete-time semi-Markov random evolutions on Banach spaces (see, e.g. [22]). The method used in the proofs is based on the martingale representation and on the convergence of transition operators of the extended SMC via singular perturbation techniques. The tightness of the family of processes is proved via Sobolev's embedding theorems and martingale representation [2], [39]. It is worth noting that, as in the Markov case, the results presented here cannot be deduced directly from the continuoustime case. Of course, alternative techniques to prove these results for the particular systems considered in this paper can be found in, e.g. [4], [15], [25], [27], [32], [35]-[37], and [38].

The results presented in this paper could also be useful for many applications where the SMC is the supporting model. For example, the additive functionals in performance analysis; the geometric Markov renewal processes in finance, insurance, and reliability; the dynamical systems in fatigue crack growth modeling [10], etc.

The paper is organized as follows. The definition and properties of discrete-time semi-Markov random evolutions (DTSMREs) are given in Section 2. Averaging, diffusion approximation, and diffusion approximation with equilibrium of DTSMREs are considered in Section 3. In Section 4 we give three applications of the above asymptotic properties: additive functionals (AFs), geometric Markov renewal chains (GMRCs), and dynamical systems. Section 5 contains the rates of convergence in the limit theorems for DTSMREs, AFs, and GMRCs. In Section 6 we present controlled DTSMREs and derive Hamilton-Jacobi-Bellman equations for them. Optimal control problems for controlled AFs and GMRCs are also studied in this section. In Section 7 we give the proofs of the theorems presented in the previous sections. In Section 8 we conclude the paper and indicate some future work.

\section{Definition and properties}

Let $\mathbb{N}$ be the set of nonnegative integer numbers, let $\mathbb{R}_{+}:=[0, \infty)$, and let $\mathcal{B}_{+}$be the Borel sets of $\mathbb{R}_{+}$. Let $(E, \mathcal{E})$ be a measurable space with a countably generated $\sigma$-algebra and $\left(\Omega, \mathcal{F},\left(\mathcal{F}_{n}\right)_{n \in \mathbb{N}}, \mathbb{P}\right)$ be a stochastic basis on which we consider a Markov renewal process $\left(x_{n}, \tau_{n}, n \in \mathbb{N}\right)$ in discrete time $k \in \mathbb{N}$, with state space $\left(E \times \mathbb{R}_{+}, \mathscr{E} \otimes \mathscr{B}_{+}\right)$. The semi-Markov kernel $q$ is defined by (see, e.g. [5] and [23])

$$
q(x, B, k):=\mathbb{P}\left(x_{n+1} \in B, \tau_{n+1}-\tau_{n}=k \mid x_{n}=x\right)
$$

for $x \in E, B \in \mathcal{E}$, and $k, n \in \mathbb{N}$. 
We will also define $q(x, B, \Gamma)=\sum_{n \in \Gamma} q(x, B, n)$, where $\Gamma \subset \mathbb{N}$. The process $\left(x_{n}\right)$ is the embedded Markov chain of the Markov renewal process $\left(x_{n}, \tau_{n}\right)$ with transition kernel $P(x, \mathrm{~d} y)$ given by $P(x, B):=q(x, B, \mathbb{N})$. The semi-Markov kernel $q$ is written as

$$
q(x, \mathrm{~d} y, k)=P(x, \mathrm{~d} y) f_{x y}(k),
$$

where $f_{x y}(k):=\mathbb{P}\left(\tau_{n+1}-\tau_{n}=k \mid x_{n}=x, x_{n+1}=y\right)$, the conditional distribution of the sojourn time in state $x$ given that the next visited state is $y$.

Define also the counting process of jumps $v_{k}=\max \left\{n: \tau_{n} \leq k\right\}$, and the discrete-time SMC $z_{k}$ by $z_{k}=x_{v_{k}}$ for $k \in \mathbb{N}$. Define the backward recurrence time process $\gamma_{k}:=k-\tau_{v_{k}}, k \geq 0$, and the filtration $\mathcal{F}_{k}:=\sigma\left(z_{\ell}, \gamma_{\ell} ; \ell \leq k\right), k \geq 0$. The process $\left(z_{k}, \gamma_{k}\right), k \geq 0$, is a Markov chain.

Let us consider a separable Banach space $\mathbb{B}$ of real-valued measurable functions defined on $E \times \mathbb{N}$, endowed with the sup norm $\|\cdot\|$, and denote by $\mathscr{B}$ its Borel $\sigma$-algebra. The Markov chain $\left(z_{k}, \gamma_{k}\right), k \geq 0$, has the following transition probability operator $P^{\sharp}$ on $\mathbb{B}$ :

$$
P^{\sharp} \varphi(x, k)=\frac{1}{\bar{H}_{x}(k)} \int_{E \backslash\{x\}} q(x, \mathrm{~d} y, k+1) \varphi(y, 0)+\frac{\bar{H}_{x}(k+1)}{\bar{H}_{x}(k)} \varphi(x, k+1) .
$$

Its stationary distribution, if it exists, is given by

$$
\pi^{\sharp}(\mathrm{d} x \times\{k\})=\frac{\rho(\mathrm{d} x) \bar{H}_{x}(k)}{m},
$$

where

$$
m:=\int_{E} \rho(\mathrm{d} x) m(x), \quad m(x)=\sum_{k \geq 0} \bar{H}_{x}(k),
$$

and $\rho(\mathrm{d} x)$ is the stationary distribution of the embedded Markov chain $\left(x_{n}\right), H_{x}(k):=q(x, E$, $[0, k])$, and $\bar{H}_{x}(k):=1-H_{x}(k)=q(x, E,[k+1, \infty))$. The probability measure $\pi$ defined by

$$
\pi(B):=\pi^{\sharp}(B \times \mathbb{N})=\int_{B} \frac{\rho(\mathrm{d} x) m(x)}{m}
$$

is the stationary probability of the $\operatorname{SMC}\left(z_{k}\right)$. It is worth noting that the existence and uniqueness of the stationary probability $\pi^{\sharp}$ is provided by the uniform ergodicity of the Markov chain $\left(z_{k}, \gamma_{k}\right)$; see below. Define the $r$ th moment of holding time in state $x \in E$ as

$$
m_{r}(x):=\sum_{k \geq 1} k^{r} q(x, E, k), \quad r=1,2, \ldots
$$

Of course, $m(x)=m_{1}(x)$ for any $x \in E$.

Define the stationary projection operator $\Pi$ on the null space of the (discrete) generating operator $Q^{\sharp}:=P^{\sharp}-I$ as

$$
\Pi \varphi(x, s)=\sum_{\ell \geq 0} \int_{E} \pi^{\sharp}(\mathrm{d} y \times\{\ell\}) \varphi(y, \ell) \mathbf{1}(x, s),
$$

where $\mathbf{1}(x, s)=1$ for any $x \in E$ and $s \in \mathbb{N}$. This operator satisfies the equation

$$
\Pi Q^{\sharp}=Q^{\sharp} \Pi=0 .
$$


The potential operator of $Q^{\sharp}$, denoted by $R_{0}$, is defined by

$$
R_{0}:=\left(Q^{\sharp}+\Pi\right)^{-1}-\Pi=\sum_{k \geq 0}\left[\left(P^{\sharp}\right)^{k}-\Pi\right] .
$$

Let us introduce a family of linear bounded contraction operators $D(x), x \in E$ (i.e. $\|D(x) \varphi\| \leq\|\varphi\|)$, defined on $\mathbb{B}$, where the maps $D(x) \varphi: E \rightarrow \mathbb{B}$ are $\mathcal{E}$-measurable and $\varphi \in \mathbb{B}$ [33]. Denote by $I$ the identity operator on $\mathbb{B}$. Let $\Pi \mathbb{B}=\mathcal{N}\left(Q^{\sharp}\right)$ be the null space, and let $(I-\Pi) \mathbb{B}=\mathcal{R}\left(Q^{\sharp}\right)$ be the range (value space) of the operator $Q^{\sharp}$. We will suppose here that the Markov chain $\left(z_{k}, \gamma_{k}, k \in \mathbb{N}\right)$ is uniformly ergodic, that is, $\sup _{\|\varphi\| \leq 1}\left\|\left(\left(P^{\sharp}\right)^{n}-\Pi\right) \varphi\right\| \rightarrow 0$ as $n \rightarrow \infty$ for any $\varphi \in \mathbb{B}$. Note that this condition implies the exponential ergodicity of the Markov chain [26]. In that case, the transition operator is reducible-invertible on $\mathbb{B}$. Thus, we have $\mathbb{B}=\mathcal{N}\left(Q^{\sharp}\right) \oplus \mathcal{R}\left(Q^{\sharp}\right)$, the direct sum of the two subspaces. The domain of an operator $A$ on $\mathbb{B}$ is denoted by $\mathscr{D}(A):=\{\varphi \in \mathbb{B}: A \varphi \in \mathbb{B}\}$.

Let us now define a DTSMRE.

Definition 2.1. A (forward) DTSMRE $\Phi_{k}, k \in \mathbb{N}$, on $\mathbb{B}$ is defined by

$$
\Phi_{k} \varphi=D\left(z_{k}\right) D\left(z_{k-1}\right) \cdots D\left(z_{2}\right) D\left(z_{1}\right) \varphi, \quad k \geq 1, \quad \Phi_{0}=I,
$$

for any $\varphi \in \mathbb{B}_{0}:=\bigcap_{x \in E} \mathscr{D}(D(x))$. Thus, we have $\Phi_{k}=D\left(z_{k}\right) \Phi_{k-1}$.

For example, consider an additive functional of the SMC $z_{k}$, i.e. $\alpha_{k}:=u+\sum_{\ell=1}^{k} a\left(z_{\ell}\right)$ for $k \geq 1$ with $\alpha_{0}=u$. Define a family of operators $D(x), x \in E$, on $\mathbb{B}$ by $D(x) \varphi(u)=$ $\varphi(u+a(x))$. Then we can write $\Phi_{k} \varphi(u)=\prod_{\ell=1}^{k} D\left(z_{\ell}\right) \varphi(u)=\varphi\left(u+\sum_{\ell=1}^{k} a\left(z_{\ell}\right)\right)=\varphi\left(z_{k}\right)$.

Remark 2.1. The important difference with the discrete-time random evolution considered in [18], [19], and [47] concerns the embedded Markov chain $x_{n}$ (of a continuous-time semiMarkov process [29], [30], [31], [34], [41]) instead of the SMC $z_{k}$, defined on the calendar time $k$, which is not Markov. Concerning the above example, for the corresponding discretetime formulation of the case referred to here, we take $\alpha_{k}:=u+\sum_{n=1}^{v_{k}} a\left(x_{n}\right)$, which is just the Markov case.

The process $M_{k}$ defined by

$$
M_{k}:=\Phi_{k}-I-\sum_{\ell=0}^{k-1} \mathbb{E}\left[\Phi_{\ell+1}-\Phi_{\ell} \mid \mathcal{F}_{\ell}\right], \quad k \geq 1, \quad M_{0}=0,
$$

on $\mathbb{B}$ is an $\mathscr{F}_{k}$-martingale. The random evolution $\Phi_{k}$ can be written as

$$
\Phi_{k}:=I+\sum_{\ell=0}^{k-1}\left[D\left(z_{\ell+1}\right)-I\right] \Phi_{\ell},
$$

and then martingale (2.3) can be written as

$$
M_{k}:=\Phi_{k}-I-\sum_{\ell=0}^{k-1} \mathbb{E}\left[\left(D\left(z_{\ell+1}\right)-I\right) \Phi_{\ell} \mid \mathcal{F}_{\ell}\right]
$$

or

$$
M_{k}:=\Phi_{k}-I-\sum_{\ell=0}^{k-1}\left(\mathbb{E}\left[D\left(z_{\ell+1}\right) \mid \mathcal{F}_{\ell}\right]-I\right) \Phi_{\ell} .
$$


Finally, as $\mathbb{E}\left[D\left(z_{\ell+1}\right) \Phi_{\ell} \varphi \mid \mathcal{F}_{\ell}\right]=\left(P^{\sharp} D(\cdot) \Phi_{\ell} \varphi\right)\left(z_{\ell}, \gamma_{\ell}\right)$, we take

$$
M_{k}:=\Phi_{k}-I-\sum_{\ell=0}^{k-1}\left[P^{\sharp} D(\cdot)-I\right] \Phi_{\ell} .
$$

\section{Average and diffusion approximation}

In this section we present average and diffusion approximation results for the DTSMRE, as well as the diffusion approximation with equilibrium.

Let $D_{\mathbb{B}}[0, \infty)$ be the Skorokhod space of $\mathbb{B}$-valued measurable functions defined on $\mathbb{R}_{+}$ which are right continuous having left-hand limits, and let $C_{\mathbb{B}}[0, \infty)$ be the $\mathbb{B}$-valued continuous functions defined on $\mathbb{R}_{+}$. On these spaces we denote by ' $\Rightarrow$ ' the weak convergence. Let $C_{0}(\mathbb{R})$ be the space of bounded continuous functions defined on $\mathbb{R}$ vanishing at $\infty$, and let $W^{l, 2}(\mathbb{R})$ be a Sobolev space.

\subsection{Averaging}

Let us set $k:=[t / \varepsilon]$, and consider the continuous-time process $M_{t}^{\varepsilon}$ :

$$
M_{t}^{\varepsilon}:=M_{[t / \varepsilon]}=\Phi_{[t / \varepsilon]}^{\varepsilon}-I-\sum_{\ell=0}^{[t / \varepsilon]-1}\left[P^{\sharp} D^{\varepsilon}(\cdot)-I\right] \Phi_{\ell}^{\varepsilon} .
$$

We will prove here asymptotic results for this process as $\varepsilon \rightarrow 0$.

The following assumptions are needed for averaging.

(A1) The Markov chain $\left(z_{k}, \gamma_{k}, k \in \mathbb{N}\right)$ is uniformly ergodic with ergodic distribution $\pi^{\sharp}(B \times$ $\{k\}), B \in \mathcal{E}$ and $k \in \mathbb{N}$.

(A2) The moments $m_{2}(x), x \in E$, are uniformly integrable.

(A3) The perturbed operators $D^{\varepsilon}(x)$ have the following representation on $\mathbb{B}$ :

$$
D^{\varepsilon}(x)=I+\varepsilon D_{1}(x)+\varepsilon D_{0}^{\varepsilon}(x) .
$$

Here the operators $D_{1}(x)$ on $\mathbb{B}$ are closed and $\mathbb{B}_{0}:=\bigcap_{x \in E} \mathscr{D}\left(D_{1}(x)\right)$ is dense in $\mathbb{B}$, $\overline{\mathbb{B}}_{0}=\mathbb{B}$. The operators $D_{0}^{\varepsilon}(x)$ are negligible, i.e. $\lim _{\varepsilon \rightarrow 0}\left\|D_{0}^{\varepsilon}(x) \varphi\right\|=0$ for any $\varphi \in \mathbb{B}_{0}$.

(A4) We have $\int_{E} \pi(\mathrm{d} x)\left\|D_{1}(x) \varphi\right\|^{2}<\infty$.

(A5) There exist Hilbert spaces $H$ and $H^{*}$ which are compactly embedded in Banach spaces $\mathbb{B}$ and $\mathbb{B}^{*}$, respectively, where $\mathbb{B}^{*}$ is a dual space to $\mathbb{B}$.

(A6) The operators $D^{\varepsilon}(x)$ and $\left(D^{\varepsilon}\right)^{*}(x)$ are contractive on Hilbert spaces $H$ and $H^{*}$, respectively.

We note that if $\mathbb{B}=C_{0}(\mathbb{R})$ then $H=W^{l, 2}(\mathbb{R})$ is a Sobolev space, and $W^{l, 2}(\mathbb{R}) \subset C_{0}(\mathbb{R})$, with this embedding compact (see [33] and [39]). For the spaces $\mathbb{B}=L_{2}(\mathbb{R})$ and $H=W^{l, 2}(\mathbb{R})$, the situation is the same.

Theorem 3.1. Under assumptions (A1)-(A6), the weak convergence

$$
\Phi_{[t / \varepsilon]}^{\varepsilon} \Rightarrow \bar{\Phi}(t) \quad \text { as } \varepsilon \rightarrow 0
$$


holds, where the limit random evolution $\bar{\Phi}(t)$ is determined by the equation

$$
\bar{\Phi}(t) \varphi-\varphi-\int_{0}^{t} \hat{\mathbb{L}} \bar{\Phi}(s) \varphi \mathrm{d} s=0, \quad 0 \leq t \leq T, \varphi \in \mathbb{B}_{0},
$$

or, equivalently,

$$
\frac{\mathrm{d}}{\mathrm{d} t} \bar{\Phi}(t) \varphi=\hat{\mathbb{L}} \bar{\Phi}(t) \varphi
$$

where the limit contracted operator is then given by

$$
\hat{\mathbb{L}}=\hat{D}_{1}=\int_{E} \pi(\mathrm{d} x) D_{1}(x) .
$$

This result generalizes the classical Krylov-Bogolyubov averaging principle [20] on Banach spaces.

\subsection{Diffusion approximation}

For the diffusion approximation, we will consider a different time scaling and some additional assumptions.

(D1) Let us assume that the perturbed operators $D^{\varepsilon}(x)$ have the following representation in $\mathbb{B}$ :

$$
D^{\varepsilon}(x)=I+\varepsilon D_{1}(x)+\varepsilon^{2} D_{2}(x)+\varepsilon^{2} D_{0}^{\varepsilon}(x) .
$$

Here the operators $D_{2}(x)$ on $\mathbb{B}$ are closed and $\mathbb{B}_{0}:=\bigcap_{x \in E} \mathscr{D}\left(D_{2}(x)\right)$ is dense in $\mathbb{B}$, $\overline{\mathbb{B}}_{0}=\mathbb{B}$; the operators $D_{0}^{\varepsilon}(x)$ are negligible operators, i.e. $\lim _{\varepsilon \rightarrow 0}\left\|D_{0}^{\varepsilon}(x) \varphi\right\|=0$.

(D2) The following balance condition holds:

$$
\Pi D_{1}(x) \Pi=0 .
$$

(D3) The moments $m_{3}(x), x \in E$, are uniformly integrable.

Theorem 3.2. Under assumptions (A1), (A5), (A6), and (D1)-(D3), the weak convergence

$$
\Phi_{\left[t / \varepsilon^{2}\right]}^{\varepsilon} \Rightarrow \Phi_{0}(t) \quad \text { as } \varepsilon \rightarrow 0
$$

holds, where the limit random evolution $\Phi_{0}(t)$ is a diffusion random evolution determined by the generator

$$
\mathbb{L}=\Pi D_{2}(x) \Pi+\Pi D_{1}(x) R_{0} D_{1}(x) \Pi-\Pi D_{1}^{2}(x) \Pi .
$$

\subsection{Diffusion approximation with equilibrium}

It is worth noting that averaged semi-Markov random evolutions can be considered as the first approximation to the initial evolutions. The diffusion approximation of the semi-Markov random evolutions determines the second approximation to the initial evolution, since the first approximation under the balance condition appears to be trivial.

Here we consider the algorithms for constructing the first and second approximations in the case when the balance condition in the diffusion approximation scheme is not fulfilled. We introduce the deviated semi-Markov random evolution as the normalized difference between the initial and averaged evolutions. In the limit, we obtain the diffusion approximation with equilibrium of the initial evolution from the averaged one.

Let us consider the DTSMRE $\Phi_{[t / \varepsilon]}^{\varepsilon}$, the averaged evolution $\bar{\Phi}(t)$ (see Section 3.1), and the deviated evolution

$$
W_{t}^{\varepsilon}:=\varepsilon^{-1 / 2}\left[\Phi_{[t / \varepsilon]}^{\varepsilon}-\bar{\Phi}(t)\right] .
$$


Theorem 3.3. Under assumptions (A1), (A5), (A6), and (D3), with operators $D^{\varepsilon}(x)$ in $(A 3)$, instead of $(D 1)$, the deviated semi-Markov random evolution $W_{t}^{\varepsilon}$ weakly converges, when $\varepsilon \rightarrow 0$, to the diffusion random evolution $W_{t}^{0}$ defined by the generator

$$
\mathbb{L}=\Pi\left(D_{1}(x)-\hat{D}_{1}\right) R_{0}\left(D_{1}(x)-\hat{D}_{1}\right) \Pi .
$$

\section{Applications on particular systems}

In this section we give three applications of the above results: (i) to additive functionals, which have many applications, e.g. in storage, reliability, and risk theories (see, e.g. [5], [18], [19], and [24]), (ii) to geometric Markov renewal processes, which also have many applications, including finance (see, e.g. [43], [44], [45], and [46]), and (iii) to dynamical systems (see, e.g. [10]).

\subsection{Additive functionals}

Let us define the following additive functional (AF):

$$
y_{k}=\sum_{l=0}^{k} a\left(z_{l}\right), \quad k \geq 0, \quad y_{0}=y .
$$

If we define the operator $D(z)$ on $C_{0}(\mathbb{R})$ by

$$
D(z) \varphi(y):=\varphi(y+a(z)),
$$

then the DTSMRE $\Phi_{k} \varphi$ has the representation

$$
\Phi_{k} \varphi(y)=\varphi\left(y_{k}\right) .
$$

Result 4.1. (Averaging of the AF.) Define the continuous-time process

$$
y_{t}^{\varepsilon}:=\varepsilon \sum_{l=0}^{[t / \varepsilon]} a\left(z_{l}\right)
$$

Then, from Theorem 3.1, it follows that this process has the weak limit $y_{0}(t)=\lim _{\varepsilon \rightarrow 0} y_{t}^{\varepsilon}$, given by

$$
y_{0}(t)=y+\hat{a} t,
$$

where $\hat{a}=\int_{E} \pi(\mathrm{d} z) a(z)$. We suppose that $\int_{E} \pi(\mathrm{d} z)|a(z)|<+\infty$.

Result 4.2. (Diffusion approximation of the AF.) Let us consider the continuous-time process

$$
\xi_{t}^{\varepsilon}:=\varepsilon \sum_{l=0}^{\left[t / \varepsilon^{2}\right]} a\left(z_{l}\right), \quad \xi_{0}^{\varepsilon}=y .
$$

Then, under the balance condition $\int_{E} \pi(\mathrm{d} z) a(z)=0$ and $\int_{E} \pi(\mathrm{d} z)|a(z)|^{2}<+\infty$, the weak limit process $\xi_{0}(t)=\lim _{\varepsilon \rightarrow 0} \xi_{t}^{\varepsilon}$ has the form

$$
\xi_{0}(t)=y+b w_{t},
$$

where $b^{2}=2 \hat{a}_{0}-\hat{a}_{2}, \hat{a}_{0}:=\sum_{\ell \geq 0} \int_{E} \pi^{\#}(\mathrm{~d} z \times\{\ell\}) a(z) R_{0} a(z), \hat{a}_{2}=\int_{E} \pi(\mathrm{d} z) a^{2}(z)$, and $w_{t}$ is a standard Wiener process. 
Result 4.3. (Diffusion approximation with equilibrium of the AF.) Let us consider the normalized additive functional

$$
w_{t}^{\varepsilon}:=\varepsilon^{-1 / 2}\left[y_{t}^{\varepsilon}-\hat{a} t\right] .
$$

Then this process converges weakly to the process $\sigma w_{t}$, where

$$
\sigma^{2}=\sum_{\ell \geq 0} \int_{E} \pi^{\#}(\mathrm{~d} z \times\{\ell\})(a(z)-\hat{a}) R_{0}(a(z)-\hat{a})
$$

and $w_{t}$ is a standard Wiener process.

In this way, the $A F y_{t}^{\varepsilon}$ may be presented in the following approximated form:

$$
y_{t}^{\varepsilon} \approx \hat{a} t+\sqrt{\varepsilon} \sigma w_{t} .
$$

\subsection{GMRCs}

The GMRC is defined as (see [43], [44], and [46])

$$
S_{k}:=S_{0} \prod_{l=1}^{k}\left(1+a\left(z_{l}\right)\right), \quad k \in \mathbb{N}, \quad S_{0}=s .
$$

We suppose that $\prod_{k=1}^{0}=1$.

If we define the operator $D(z)$ on $C_{0}(\mathbb{R})$ by

$$
D(z) \varphi(s):=\varphi(s(1+a(z))),
$$

then the DTSMRE $\Phi_{k} \varphi$ has the representation

$$
\Phi_{k} \varphi(s)=\varphi\left(S_{k}\right) .
$$

It is worth noting here that we are using $S_{t}^{\varepsilon}$ instead of $\ln S_{t}^{\varepsilon}$ in order to be consistent with the discrete models for stock prices in mathematical finance proposed by Cox et al. [11] and Aase [1].

Result 4.4. (Averaging of the GMRC.) Define the following sequence of processes:

$$
S_{t}^{\varepsilon}:=S_{0} \prod_{k=1}^{[t / \varepsilon]}\left(1+\varepsilon a\left(z_{k}\right)\right), \quad t \in \mathbb{R}_{+}, \quad S_{0}=s .
$$

Then, under averaging conditions, the limit process $\bar{S}_{t}$ has the form

$$
\bar{S}_{t}=s \mathrm{e}^{\hat{a} t}
$$

where $\hat{a}=\int_{E} \pi(\mathrm{d} z) a(z)$.

Result 4.5. (Diffusion approximation of the GMRC.) Define the following sequence of processes:

$$
S^{\varepsilon}(t):=S_{0} \prod_{k=1}^{\left[t / \varepsilon^{2}\right]}\left(1+\varepsilon a\left(z_{k}\right)\right), \quad t \in \mathbb{R}_{+}, \quad S_{0}=s .
$$

Then in the diffusion approximation scheme, we have the limit process

$$
S_{0}(t)=s \mathrm{e}^{-t \hat{a}_{2} / 2} \mathrm{e}^{\sigma_{a} w(t)},
$$


where

$$
\hat{a}_{2}:=\int_{E} \pi(\mathrm{d} z) a^{2}(z), \quad \sigma_{a}^{2}:=\frac{1}{2} \int_{E} \pi(\mathrm{d} z) a^{2}(z)+\sum_{\ell \geq 0} \int_{E} \pi^{\#}(\mathrm{~d} z \times\{\ell\}) a(z) R_{0} a(z) .
$$

This means that $S_{0}(t)$ satisfies the stochastic differential equation

$$
\frac{\mathrm{d} S_{0}(t)}{S_{0}(t)}=\frac{1}{2}\left(\sigma_{a}^{2}-\hat{a}_{2}\right) \mathrm{d} t+\sigma_{a} \mathrm{~d} w_{t}
$$

where $w_{t}$ is a standard Wiener process.

Result 4.6. (Diffusion approximation with equilibrium of the GMRC.) Let us consider the normalized GMRC

$$
w_{t}^{\varepsilon}:=\varepsilon^{-1 / 2}\left[\ln \left(\frac{S_{t}^{\varepsilon}}{S_{0}}\right)-\hat{a} t\right] .
$$

It is worth noting that in finance the expression $\ln \left(S_{t}^{\varepsilon} / S_{0}\right)$ represents the log-return of the underlying asset (stock for example) $S_{t}^{\varepsilon}$.

Then this process converges weakly to the process $\sigma w_{t}$, where

$$
\sigma^{2}=\int_{E} \pi(\mathrm{d} z)(a(z)-\hat{a}) R_{0}(a(z)-\hat{a})
$$

and $w_{t}$ is a standard Wiener process.

In this way, the GMRC $S_{t}^{\varepsilon}$ may be presented in the following approximated form:

$$
S_{t}^{\varepsilon} \approx S_{0} \mathrm{e}^{\hat{a} t+\sqrt{\varepsilon} \sigma w_{t}}
$$

\subsection{Dynamical systems}

We consider here discrete-time dynamical systems (DSs) and their asymptotic behavior in the series scheme: the average and diffusion approximation (see [23]).

Let us consider the difference equation

$$
y_{k+1}^{\varepsilon}=y_{k}^{\varepsilon}+\varepsilon C\left(y_{k}^{\varepsilon} ; z_{k+1}\right), \quad k \geq 0, \quad y_{0}^{\varepsilon}=u,
$$

switched by the SMC $\left(z_{k}\right)$.

The perturbed operators $D^{\varepsilon}(x), x \in E$, are now defined by

$$
D^{\varepsilon}(x) \varphi(u)=\varphi(u+\varepsilon C(u, x)) .
$$

Result 4.7. (Averaging of the DS.) Under averaging assumptions, the weak convergence

$$
y_{[t / \varepsilon]}^{\varepsilon} \Rightarrow \bar{y}(t) \quad \text { as } \varepsilon \rightarrow 0
$$

holds, where $\bar{y}(t), t \geq 0$, is the solution of the (deterministic) differential equation

$$
\frac{\mathrm{d}}{\mathrm{d} t} \bar{y}(t)=\bar{C}(\bar{y}(t)) \text { and } \bar{y}(0)=u,
$$

where $\bar{C}(u)=\int_{E} \pi(\mathrm{d} x) C(u, \mathrm{~d} x)$. 
Result 4.8. (Diffusion approximation of the DS.) Under diffusion approximation conditions, the weak convergence

$$
y_{\left[t / \varepsilon^{2}\right]}^{\varepsilon} \Rightarrow x_{t} \quad \text { as } \varepsilon \rightarrow 0
$$

holds, where $x_{t}, t \geq 0$, is a diffusion processes with initial value $x_{0}=u$, determined by the operator

$$
\mathbb{L} \varphi(u)=a(u) \varphi^{\prime}(u)+\frac{1}{2} b^{2}(u) \varphi^{\prime \prime}(u),
$$

provided that $b^{2}(u)>0$, and drift and diffusion coefficients are defined as

$$
b^{2}(u):=2 \bar{C}_{0}(u)-\bar{C}_{2}(u), \quad a(u):=\bar{C}_{01}(u)-\bar{C}_{1}(u),
$$

with

$$
\begin{array}{rlrl}
\bar{C}_{0}(u) & :=\sum_{\ell \geq 0} \int_{E} \pi^{\#}(\mathrm{~d} x \times\{\ell\}) C_{0}(u, x), & & C_{0}(u, x):=C(u, x) R_{0} C(u, x), \\
\bar{C}_{01}(u) & :=\sum_{\ell \geq 0} \int_{E} \pi^{\#}(\mathrm{~d} x \times\{\ell\}) C_{01}(u, x), & C_{01}(u, x):=C(u, x) R_{0} C_{u}^{\prime}(u, x), \\
\bar{C}_{1}(u):=\int_{E} \pi(\mathrm{d} x) C_{1}(u, x), & & C_{1}(u, x):=C(u, x) C_{u}^{\prime}(u, x), \\
\bar{C}_{2}(u) & :=\int_{E} \pi(\mathrm{d} x) C^{2}(u, x) . &
\end{array}
$$

\section{Rates of convergence in the limit theorems}

In this section we present the rates of convergence of the DTSMRE in the averaging, diffusion approximation, and diffusion approximation with equilibrium schemes ((A1)-(A3)), and we give the rates of convergence for AFs and GMRCs in the corresponding limits as corollaries.

Proposition 5.1. The rate of convergence of the DTSMRE in the averaging scheme has the form

$$
\left\|\mathbb{E}\left[\Phi_{[t / \varepsilon]}^{\varepsilon} \varphi\right]-\bar{\Phi}(t) \varphi\right\| \leq \varepsilon A\left(T, \varphi,\left\|R_{0}\right\|,\left\|D_{1}\right\|\right),
$$

where $A\left(T, \varphi,\left\|R_{0}\right\|,\left\|D_{1}\right\|\right)$ is a constant and $0 \leq t \leq T$.

The proof of this proposition is given in Section 7.4.

Proposition 5.2. The rate of convergence of the DTSMRE in the diffusion approximation scheme takes the form

$$
\left\|\mathbb{E}\left[\Phi_{\left[t / \varepsilon^{2}\right]}^{\varepsilon} \varphi\right]-\Phi_{0}(t) \varphi\right\| \leq \varepsilon D\left(T, \varphi,\left\|R_{0}\right\|,\left\|D_{1}\right\|,\left\|D_{2}\right\|\right),
$$

where $D\left(T, \varphi,\left\|R_{0}\right\|,\left\|D_{1}\right\|,\left\|D_{2}\right\|\right)$ is a constant and $0 \leq t \leq T$.

Proposition 5.3. The rate of convergence of the DTSMRE in the diffusion approximation with equilibrium scheme has the form

$$
\left\|\mathbb{E}\left[W_{t}^{\varepsilon} \varphi\right]-W_{t}^{0} \varphi\right\| \leq \sqrt{\varepsilon} N\left(T, \varphi,\left\|R_{0}\right\|,\left\|D_{1}\right\|,\left\|D_{1}^{2}\right\|\right),
$$

where $N\left(T, \varphi,\left\|R_{0}\right\|,\left\|D_{1}\right\|,\left\|D_{1}^{2}\right\|\right)$ is a constant and $0 \leq t \leq T$. 
The proofs of Propositions 5.2 and 5.3 are similar to that of Proposition 5.1. In Corollaries 5.1 and 5.2 below we give some rate of convergence results concerning applications to particular systems.

Corollary 5.1. (i) The rate of convergence of the AF in the averaging scheme has the form

$$
\left\|\mathbb{E} y_{t}^{\varepsilon}-y_{0}(t)\right\| \leq \varepsilon a\left(T,\left\|R_{0}\right\|, \kappa_{1}\right),
$$

where $a\left(T,\left\|R_{0}\right\|, \kappa_{1}\right)$ is a constant, with $\kappa_{1}:=\sup _{x \in E}|a(x)|$, and $0 \leq t \leq T$.

(ii) The rate of convergence of the AF in the diffusion approximation scheme has the form

$$
\left\|\mathbb{E} \xi_{t}^{\varepsilon}-\xi_{0}(t)\right\| \leq \varepsilon d\left(T,\left\|R_{0}\right\|, \kappa_{1}, \kappa_{2}\right)
$$

where $d\left(T,\left\|R_{0}\right\|, \kappa_{1}, \kappa_{2}\right)$, with $\kappa_{2}:=\sup _{x \in E}\left|a^{2}(x)\right|$ is a constant, and $0 \leq t \leq T$.

(iii) The rate of convergence of the $A F$ in the diffusion approximation with equilibrium scheme has the form

$$
\left\|\mathbb{E} W_{t}^{\varepsilon}-w_{t}\right\| \leq \sqrt{\varepsilon} n\left(T,\left\|R_{0}\right\|, \kappa_{1}, \kappa_{2}\right),
$$

where $n\left(T,\left\|R_{0}\right\|, \kappa_{1}, \kappa_{2}\right)$ is a constant and $0 \leq t \leq T$.

Corollary 5.2. (i) The rate of convergence of the GMRC in the averaging scheme has the form

$$
\left\|\mathbb{E} S_{t}^{\varepsilon}-\bar{S}_{t}\right\| \leq \varepsilon a\left(T,\left\|R_{0}\right\|, \kappa_{1}\right),
$$

where $a\left(T,\left\|R_{0}\right\|, \kappa_{1}\right)$ is a constant and $0 \leq t \leq T$.

(ii) The rate of convergence of the GMRC in the diffusion approximation scheme has the form

$$
\left.\| \mathbb{E} S_{t}^{\varepsilon}-S_{0}(t)\right) \| \leq \varepsilon d\left(T,\left\|R_{0}\right\|, \kappa_{1}, \kappa_{2}\right),
$$

where $d\left(T,\left\|R_{0}\right\|, \kappa_{1}, \kappa_{2}\right)$ is a constant and $0 \leq t \leq T$.

(iii) The rate of convergence of the GMRC in the diffusion approximation with equilibrium scheme has the form

$$
\left\|\mathbb{E} W_{t}^{\varepsilon}-\sigma w_{t}\right\| \leq \sqrt{\varepsilon} n\left(T,\left\|R_{0}\right\|, \kappa_{1}, \kappa_{2}\right),
$$

where $n\left(T,\left\|R_{0}\right\|, \kappa_{1}, \kappa_{2}\right)$ is a constant and $0 \leq t \leq T$.

\section{Optimal control and the Hamilton-Jacobi-Bellman equation for DTSMREs}

In this section we consider the optimal control of the DTSMRE and derive the HamiltonJacobi-Bellman (HJB) equation in this case. The derivation is based on Dynkin's formula and the boundary value problem for random evolutions (see [42]). We also present examples of Dynkin's formulae and boundary value problems for controlled additive functionals (CAFs) and controlled geometric Markov renewal chains (CGMRCs). In the literature a CAF is usually called the Markov decision process (see, for example, [3], [7], [8], and [9]). We also mention that the discrete-time case of the stochastic optimal control has been considered in [6]. 


\subsection{Controlled DTSMREs and Dynkin's formula, and their boundary value problem}

Let $U$ denote a compact Polish space representing the control, and let $u_{k}$ be a $U$-valued control process, which we suppose to be a Markov chain. We note that we can define the process $u_{v_{k}}$ which is a semi-Markov control process, considered in many papers (see, e.g. [16] and [48]).

Definition 6.1. A controlled DTSMRE (CDTSMRE) $\Phi_{k}^{u}, u \in U$ and $k \in \mathbb{N}$, on $\mathbb{B}$, is defined by

$$
\Phi_{k}^{u} \varphi=D\left(z_{k}, u_{k}\right) D\left(z_{k-1}, u_{k-1}\right) \cdots D\left(z_{2}, u_{2}\right) D\left(z_{1}, u_{1}\right) \varphi
$$

for $k \geq 1, \Phi_{0}^{u}=I, u_{0}=u \in U$, and any $\varphi \in \mathbb{B}_{0}:=\bigcap_{x \in E, u \in U} \mathcal{D}(D(x, u))$. Thus, we have $\Phi_{k}=D\left(z_{k}, u_{k}\right) \Phi_{k-1}$.

We note that the process $\left(z_{k}, \gamma_{k}, u_{k}\right)$ is a Markov chain on $E \times \mathbb{R}_{+} \times U$ adapted to the filtration $\mathscr{F}_{k}:=\sigma\left(z_{\ell}, \gamma_{\ell}, u_{\ell} ; \ell \leq k\right), k \geq 0$. We also note that $\left(\Phi_{k}^{u} \varphi, z_{k}, \gamma_{k}, u_{k}\right)$ is a Markov chain on $\mathbb{B} \times E \times \mathbb{R}_{+} \times U$.

Example 6.1. (CAF or Markov decision chain.) Let us define the following CAF:

$$
y_{k}^{u}=\sum_{l=0}^{k} a\left(z_{l}, u_{l}\right), \quad k \geq 0, \quad y_{0}=y .
$$

If we define the operator $D(z, u)$ on $C_{0}(\mathbb{R})$ by

$$
D(z, u) \varphi(y):=\varphi(y+a(z, u)),
$$

then the CDTSMRE $\Phi_{k}^{u} \varphi$ has the form

$$
\Phi_{k}^{u} \varphi(y)=\varphi\left(y_{k}^{u}\right) .
$$

Example 6.2. (CGMRC.) The CGMRC is defined as

$$
S_{k}^{u}:=S_{0} \prod_{l=1}^{k}\left(1+a\left(z_{l}, u_{l}\right)\right), \quad k \in \mathbb{N}, \quad S_{0}=s .
$$

We suppose that $\prod_{k=1}^{0}=1$.

If we define the operator $D(z, u)$ on $C_{0}(\mathbb{R})$ by

$$
D(z, u) \varphi(s):=\varphi(s(1+a(z, u))),
$$

then the CDTSMRE $\Phi_{k}^{u} \varphi$ has the form

$$
\Phi_{k}^{u} \varphi(s)=\varphi\left(S_{k}^{u}\right)
$$

To the best of the authors' knowledge, this process has not been defined in the literature and the notion of CGMRCs is new.

Let us define the following processes: $z_{k}:=z_{v_{k}}, \gamma_{k}:=k-\tau_{\nu_{k}}$, and $\Phi_{k}^{u} \varphi:=\Phi_{v_{k}}^{u} \varphi$ (see Section 2). We also use the notation

$$
y_{k}:=\left(z_{k}, \gamma_{k}\right), \quad y_{0}:=y=(z, 0) .
$$


The following process $\left(\Phi_{k}^{u} \varphi, z_{k}, \gamma_{k}\right)$ is a Markov process on $\mathbb{B} \times E \times U$ with infinitesimal generator:

$$
L^{u} \varphi=P^{\sharp} \varphi+\int_{E} P^{\sharp}(z, \mathrm{~d} v)[D(v, u)-I] \varphi .
$$

Here $P^{\sharp}$ is the transition operator defined in (2.1).

The following two results follow from general results for multiplicative operator functionals (MOFs) (it is easy to understand from the definition that the DTSMRE is a MOF) and the proofs may be found in [42].

Result 6.1. (Dynkin's formula for the CDTSMRE.) Let $\tau$ be a stopping time such that $\mathbb{E} \tau<+\infty$. Then the following formula is valid for the CDTSMRE:

$$
\begin{aligned}
\mathbb{E}_{y, u}\left[\Phi_{\tau}^{u} \varphi\left(y_{\tau}, \tau\right)\right]= & \varphi(y, k) \\
& +\mathbb{E}_{y, u, k}\left[\sum_{i=k}^{\tau} \Phi_{i}^{u}\left\{P^{\sharp}+\int_{E} P^{\sharp}(z, \mathrm{~d} v)\left[D\left(v, u_{i}\right)-I\right] \varphi\left(y_{i}, i\right)\right\}\right] .
\end{aligned}
$$

Example 6.3. (Dynkin's formula for the CAF.) From Example 6.1 and Dynkin's formula for the CDTSMRE, it follows that Dynkin's formula for the CAF is given by

$$
\begin{aligned}
& \mathbb{E}_{y, z, u, k}\left[f\left(y_{\tau}^{u}, z_{\tau}, \tau\right)\right]-f(y, z, k) \\
& \quad=\mathbb{E}_{y, z, u, k} \sum_{i=k}^{\tau}\left\{P^{\sharp} f\left(y_{i}^{u}, z_{i}, i\right)+\int_{E} P^{\sharp}(z, \mathrm{~d} z)\left[f\left(y_{i}^{u}+a\left(z, u_{i}\right), z, i\right)-f\left(y_{i}, z, i\right)\right]\right\},
\end{aligned}
$$

where $\tau$ is a stopping time such that $\mathbb{E}_{y, z, u} \tau<+\infty$.

Example 6.4. (Dynkin's formula for the CGMRC.) From Example 6.2 and Dynkin's formula for the CDTSMRE, it follows that Dynkin's formula for the CGMRC is given by

$$
\begin{aligned}
& \mathbb{E}_{s, z, u, k}\left[f\left(S_{\tau}^{u}, z_{\tau}, \tau\right)\right]-f(s, z, k) \\
& \quad=\mathbb{E}_{s, z, u, k} \sum_{i=k}^{\tau}\left\{P^{\sharp} f\left(S_{i}^{u}, z_{i}, i\right)+\int_{E} P^{\sharp}(z, \mathrm{~d} z)\left[f\left(S_{i}\left(1+a\left(z, u_{i}\right)\right), z_{i}, i\right)-f\left(S_{i}^{u}, z_{i}, i\right)\right]\right\},
\end{aligned}
$$

where $S_{0}^{u}:=s$.

Result 6.2. (Boundary value problem for the CDTSMRE.) The solution of the CDTSMRE boundary value problem (BVP)

$$
\begin{gathered}
P^{\sharp} H(\varphi, z, u, k)+\int_{E} P^{\sharp}(x, \mathrm{~d} v)[D(v, u)-I] H(\varphi, z, u, k)=-K(\varphi, z, u, k), \quad y \in G^{0}, \\
\left.H(\varphi, z, u, k)\right|_{\partial \bar{G}}=b(y, z, k),
\end{gathered}
$$

where $G$ is a closed set with interior $G^{0}$ and boundary $\partial G$, is given by

$$
H(\varphi, z, u, k)=\mathbb{E}_{\varphi, z, u, k} \sum_{i=k}^{\tau_{G}} K\left(\Phi_{i}^{u} \varphi, z_{i}, u_{i}, i\right)+\mathbb{E}_{\varphi, z, u, k}\left[b\left(\Phi_{\tau_{G}}^{u} \varphi, z_{\tau_{G}}, \tau_{G}\right)\right],
$$

where $\tau_{G}:=\inf \left\{i: y_{i} \notin G\right\}$. 
Example 6.5. (BVP for the CAF.) The solution of the BVP for the CAF

$$
\begin{aligned}
& P^{\sharp} H(y, z, u, k)+\int_{E} P^{\sharp}(z, \mathrm{~d} v)[H(y+a(v, u), v, u, k)-H(y, v, u, k)] \\
& \quad=-K(y, z, u, k), \quad y \in G^{0}, \\
& \left.H(y, z, u, k)\right|_{\partial \bar{G}}=b(y, z, k),
\end{aligned}
$$

is given by

$$
H(y, z, u, k)=\mathbb{E}_{y, u, k} \sum_{i=k}^{\tau_{G}} K\left(y_{i}, z_{i}, u_{i}, i\right)+\mathbb{E}_{y, u, k}\left[b\left(y_{\tau_{G}}, z_{\tau_{G}}, u_{\tau_{G}}, \tau_{G}\right)\right] .
$$

Example 6.6. (BVP for the CGMRC.) The solution of the BVP for the CGMRC

$$
\begin{aligned}
& P^{\sharp} H(s, z, u, k)+\int_{E} P^{\sharp}(z, \mathrm{~d} v)[H(s(1+a(v, u)), v, u, t)-H(s, v, u, k)] \\
& \quad=-K(s, z, u, k), \quad y \in G^{0}, \\
& \left.H(y, z, u, k)\right|_{\partial \bar{G}}=b(y, z, k),
\end{aligned}
$$

is given by

$$
H(y, z, u, k)=\mathbb{E}_{y, u, k} \sum_{i=k}^{\tau_{G}} K\left(S_{i}^{u}, z_{i}, u_{i}, i\right)+\mathbb{E}_{y, u, k}\left[b\left(S_{\tau_{G}}^{u}, z_{\tau_{G}}, u_{\tau_{G}}\right)\right] .
$$

\subsection{Optimal control of the CDTSMRE}

Let $G$ be a compact set in $\mathbb{B}$ with a smooth boundary $\partial G$. Let $G^{0}$ denote the interior of $G$, and suppose that $G$ is the closure of its interior. Define the stopping time $\tau_{G}$ by

$$
\tau_{G}:=\inf \left\{k: \Phi_{k}^{u} \varphi \notin G^{0}\right\} .
$$

We suppose that $\mathbb{E} \tau_{G}<+\infty$.

Also, let $K(\varphi, z, u, k)$ and $b(\varphi, z, k)$ be two real-valued bounded functions on $\mathbb{B} \times E \times U \times \mathbb{R}_{+}$ and $\mathbb{B} \times U \times \mathbb{R}_{+}$, respectively. Let the cost functional for the CDTSMRE be defined as

$$
C(\varphi, z, u, k):=\mathbb{E}_{z, k}\left[\sum_{i=k}^{\tau_{G}} K\left(\Phi_{i}^{u} \varphi, z_{i}, u_{i}, i\right)+b\left(\Phi_{\tau_{G}}^{u} \varphi, z_{\tau_{G}}, \tau_{G}\right)\right],
$$

and let the optimal cost function for the CDTSMRE be defined as

$$
C_{0}(\varphi, z, k):=\inf _{u} C(\varphi, z, u, k) .
$$

We call the functions $K(\varphi, z, u, k)$ and $b(\varphi, z, k)$ the current payments (or current cost) and the final payments (or final cost) functions, respectively.

Theorem 6.1. The optimal cost function satisfies the equation (we call it the HJB equation for the CDTSMRE)

$$
\begin{gathered}
\inf _{u}\left[L^{u} C_{0}(\varphi, z, k)+K(\varphi, z, u, k)\right]=0, \quad \varphi \in G^{0}, \\
C_{0}(\varphi, z, k)=b(\varphi, z, k), \quad \varphi \in \partial G,
\end{gathered}
$$

where the operator $L^{u}$ is as defined in (6.1).

The proof of this theorem follows from the BVP for the CDTSMRE (see Section 7.5). 


\subsection{Optimal control of the CAF and CGMRC}

We present here the HJB equations for the CAF and CGMRC. These results follow from Section 5.1.

6.3.1. HJB equation for the CAF. The optimal cost function $C_{0}(y, z, t)$ for the CAF satisfies the HJB equation

$$
\begin{gathered}
\inf _{u}\left[L^{u} C_{0}(y, z, t)+K(y, z, u, t)\right]=0, \quad \varphi \in G^{0}, \\
C_{0}(y, z, t)=b(y, z, t), \quad \varphi \in \partial G,
\end{gathered}
$$

where the operator $L^{u}$ is defined as

$$
L^{u} f(y, z, t)=P^{\sharp} f(y, z, t)+\left[P^{\sharp} f(y+a(z, u), z, t)-f(y, z, t)\right]
$$

and $P^{\sharp}$ is as defined in (2.1).

6.3.2. HJB equation for the CGMRC. The optimal cost function $C_{0}(s, z, t)$ for the CGMRC satisfies the HJB equation

$$
\begin{gathered}
\inf _{u}\left[L^{u} C_{0}(s, z, t)+K(s, z, u, t)\right]=0, \quad \varphi \in G^{0}, \\
C_{0}(s, z, t)=b(s, z, t), \quad \varphi \in \partial G,
\end{gathered}
$$

where the operator $L^{u}$ is defined as

$$
L^{u} f(s, z, t)=P^{\sharp} f(s, z, t)+\left[P^{\sharp} f(s(1+a(z, u)), z, t)-f(s, z, t)\right]
$$

and $P^{\sharp}$ is as defined in $(2.1), s:=S_{0}$.

\section{Proofs}

In this section we prove the weak convergence limit theorems via the martingale representation and singular perturbing problems in two steps. In the first step, we prove the relative compactness of the family of processes by establishing the compact containment condition and then the relative compactness of $\ell\left(\Phi_{t}^{\varepsilon}\right)$, where $\ell \in \mathbb{B}^{*}$, a dual space of $\mathbb{B}$ which separates points. In the second step, we prove the convergence of the perturbed transition operator of the extended semi-Markov renewal chains.

\subsection{Proof of Theorem 3.1}

We first prove the relative compactness of the DTSMRE in the average scheme. Let us consider the DTSMRE $\Phi_{[t / \varepsilon]}^{\varepsilon} \varphi$, as defined by relation (2.2) which is weakly compact in $D_{\mathbb{B}}[0, \infty)$ with limit points into $C_{\mathbb{B}}[0, \infty)$, the space of $\mathbb{B}$-valued continuous functions defined on $[0, \infty)$. The proof is based on the following lemmas.

Lemma 7.1. Under the conditions of Theorem 3.1, the family of processes $\Phi_{[t / \varepsilon]}^{\varepsilon} \varphi, \varphi \in \mathbb{B}_{0}$, satisfies the compact containment condition, and its limit points, as $\varepsilon \rightarrow 0$, belong to $C_{\mathbb{B}}[0, \infty)$.

Proof. We note that, from conditions (A5) and (A6), it follows that the DTSMRE $\Phi_{k} \varphi$ is a contractive operator in $H$ and, hence, $\left\|\Phi_{k} \varphi\right\|_{H}$ is a supermartingale for any $\varphi \in H$, where $\|\cdot\|_{H}$ is a norm in the Hilbert space $H$ (see [19] and [23]). Obviously, the same properties satisfy the family $\Phi_{[t / \varepsilon]}^{\varepsilon}$. Using Doob's inequality for the supermartingale $\left\|\Phi_{[t / \varepsilon]}^{\varepsilon}\right\|_{H}$, we obtain

$$
\mathbb{P}\left\{\Phi_{[t / \varepsilon]}^{\varepsilon} \varphi \in K_{\Delta}\right\} \geq 1-\Delta,
$$


where $K_{\Delta}$ is a compact set in $\mathbb{B}$ and $\Delta$ is any small number. This means that the sequence $\Phi_{[t / \varepsilon]}^{\varepsilon} \varphi$ satisfies the compact containment condition in $\mathbb{B}$. Taking into account assumptions (A1)-(A6), we find that the DTSMRE $\Phi_{[t / \varepsilon]}^{\varepsilon}$ is weakly compact in $D_{\mathbb{B}}[0,+\infty)$ with limit points in $C_{\mathbb{B}}[0,+\infty), \varphi \in \mathbb{B}_{0}$.

Let

$$
J_{t}^{\varepsilon}:=J\left(\Phi_{[t / \varepsilon]}^{\varepsilon} ;\left[\begin{array}{c}
t \\
\varepsilon
\end{array}\right]\right):=\sup _{k \leq[t / \varepsilon]}\left\|\Phi_{[t / \varepsilon]+k}^{\varepsilon} \varphi-\Phi_{[t / \varepsilon]}^{\varepsilon} \varphi\right\|,
$$

and let $K_{\Delta}$ be a compact set of $\mathbb{B}$ but with the compact containment condition given in the relation above. That is, $\Delta$ gives the bound $1-\Delta$. It is sufficient to show that $J_{t}^{\varepsilon}$ weakly converges to 0 . This is equivalent to the convergence of $J_{t}^{\varepsilon}$ in probability as $\varepsilon \rightarrow 0$.

From the very definition of $J_{t}^{\varepsilon}$ and (A3), we obtain

$$
J_{t}^{\varepsilon} \mathbf{1}_{K_{\Delta}} \leq \varepsilon \sup _{k \leq[t / \varepsilon]} \sup _{\varphi \in S_{\Delta}}\left(\left\|D_{1}\left(z_{k}\right) \varphi\right\|+\left\|D_{0}^{\varepsilon}\left(z_{k}\right) \varphi\right\|\right)
$$

where $\mathbf{1}_{K_{\Delta}}$ is the indicator of the set $K_{\Delta}$, and $S_{\Delta}$ is the finite $\delta$-set for $K_{\Delta}$. Then, for $\delta<\Delta$, we have

$$
\begin{aligned}
\mathbb{P}_{\pi}\left(J_{t}^{\varepsilon} \mathbf{1}_{K_{\Delta}}>\Delta\right) & \leq \mathbb{P}_{\pi}\left(\sup _{k \leq[t / \varepsilon]} D_{k}>\frac{\Delta-\delta}{\varepsilon}\right) \\
& =\sum_{i=1}^{[t / \varepsilon]} \mathbb{P}_{\pi}\left(\left\{\sup _{k \leq[t / \varepsilon]} D_{k}>\frac{\Delta-\delta}{\varepsilon}\right\} \bigcap D_{i}\right) \\
& \leq \varepsilon^{2}\left[\begin{array}{l}
t \\
\varepsilon
\end{array}\right] \sup _{\varphi \in S_{\Delta}}\left[( P ^ { \sharp } ) ^ { [ t / \varepsilon ] } \left(\left\|D_{1}(x) \varphi\right\|^{2}+2\left\|D_{1}(x) \varphi\right\|\left\|D_{0}^{\varepsilon}(x) \varphi\right\|\right.\right. \\
& \left.\left.+\left\|D_{0}^{\varepsilon}(x) \varphi\right\|^{2}\right)\right],
\end{aligned}
$$

where $D_{k}:=\sup _{\varphi \in S_{\Delta}}\left(\left\|D_{1}\left(z_{k}\right) \varphi\right\|+\left\|D_{0}^{\varepsilon}\left(z_{k}\right) \varphi\right\|\right)$ and

$$
D_{i}:=\left\{\omega: D_{k} \text { attains the maximum for the first time on the variable } D_{i}\right\} .
$$

It is worth noting that the operator $\left(P^{\sharp}\right)^{k}$ is bounded when $k \rightarrow \infty$. This is also the case for $\left(P^{\sharp}\right)^{[t / \varepsilon]}$ when $\varepsilon \rightarrow 0$.

Letting both $\varepsilon$ and $\delta$ go to 0 gives the result of the lemma.

Let us now consider the continuous-time martingale

$$
M_{t}^{\varepsilon}:=M_{[t / \varepsilon]}^{\varepsilon}=\Phi_{[t / \varepsilon]}^{\varepsilon}-I-\sum_{k=0}^{[t / \varepsilon]-1} \mathbb{E}_{\pi}\left[\Phi_{k+1}^{\varepsilon}-\Phi_{k}^{\varepsilon} \mid \mathcal{F}_{k}\right]
$$

Lemma 7.2. The process

$$
M_{t}^{\varepsilon}:=\Phi_{[t / \varepsilon]}^{\varepsilon}-I-\sum_{\ell=0}^{[t / \varepsilon]-1}\left[P^{\sharp} D^{\varepsilon}(\cdot)-I\right] \Phi_{\ell}^{\varepsilon}
$$

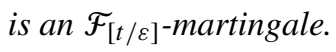


Proof. As long as

$$
M_{k}^{\varepsilon}:=\Phi_{k}^{\varepsilon}-I-\sum_{\ell=0}^{k-1}\left[P^{\sharp} D^{\varepsilon}(\cdot)-I\right] \Phi_{\ell}^{\varepsilon}
$$

is an $\mathcal{F}_{k}$-martingale, $M_{t}^{\varepsilon}=M_{[t / \varepsilon]}^{\varepsilon}$ is an $\mathcal{F}_{[t / \varepsilon]}$-martingale. Here we have $\mathbb{E}_{\pi}\left[M_{k+1}^{\varepsilon} \mid \mathcal{F}_{k}\right]=M_{k}^{\varepsilon}$, which can be easily checked.

Lemma 7.3. The family $\ell\left(\sum_{k=0}^{[t / \varepsilon]} \mathbb{E}_{\pi}\left[\Phi_{k+1}^{\varepsilon} \varphi-\Phi_{k}^{\varepsilon} \varphi \mid \mathcal{F}_{k}\right]\right)$ is relatively compact for all $\ell \in \mathbb{B}_{0}^{*}$, the dual of the space $\mathbb{B}_{0}$.

Proof. Let

$$
N_{t}^{\varepsilon}:=\sum_{k=0}^{[t / \varepsilon]} \mathbb{E}_{\pi}\left[\left(\Phi_{k+1}^{\varepsilon}-\Phi_{k}^{\varepsilon}\right) \varphi \mid \mathcal{F}_{k}\right]
$$

Then

$$
N_{t}^{\varepsilon}=\sum_{k=0}^{[t / \varepsilon]}\left[P^{\sharp} D^{\varepsilon}(\cdot)-I\right] \Phi_{k}^{\varepsilon} \varphi .
$$

As long as $\Phi_{k+1}^{\varepsilon}=D^{\varepsilon}\left(z_{k+1}\right) \Phi_{k}^{\varepsilon}$, we obtain

$$
\mathbb{E}_{\pi}\left[\Phi_{k+1}^{\varepsilon} \varphi \mid \mathcal{F}_{k}\right]=\mathbb{E}_{\pi}\left[D^{\varepsilon}\left(z_{k+1}\right) \Phi_{k}^{\varepsilon} \varphi \mid \mathcal{F}_{k}\right]
$$

Then

$$
\begin{aligned}
\left|\ell\left(\sum_{k=[t / \varepsilon]+1}^{[(t+\eta) / \varepsilon]} \mathbb{E}_{\pi}\left[\Phi_{k+1}^{\varepsilon} \varphi-\Phi_{k}^{\varepsilon} \varphi \mid \mathcal{F}_{k}\right]\right)\right|= & \ell\left(\sum_{k=[t / \varepsilon]+1}^{[(t+\eta) / \varepsilon]}\left[P^{\sharp} D^{\varepsilon}\left(z_{k+1}\right)-I\right] \Phi_{k}^{\varepsilon} \varphi\right) \mid \\
\leq & \varepsilon\|\ell\|\left(\left[\frac{t+\eta}{\varepsilon}\right]-\left[\frac{t}{\varepsilon}\right]-1\right) \\
& \times\left\|P^{\sharp}\left(D_{1}\left(z_{k+1}\right)+D_{0}^{\varepsilon}\left(z_{k+1}\right)\right) \varphi\right\| \\
\leq & \varepsilon\|\ell\| \frac{\eta}{\varepsilon}\left\|P^{\sharp}\left(D_{1}(\cdot)+D_{0}^{\varepsilon}(\cdot)\right) \varphi\right\| \\
= & \eta\|\ell\|\left\|P^{\sharp}\left(D_{1}(\cdot)+D_{0}^{\varepsilon}(\cdot)\right) \varphi\right\| \\
& \rightarrow 0 \quad \text { as } \eta \rightarrow 0,
\end{aligned}
$$

since $\left\|P^{\sharp}\left(D_{1}(\cdot)+D_{0}^{\varepsilon}(\cdot)\right) \varphi\right\|$ is bounded for any $\varphi \in \mathbb{B}_{0}$. Thus, the family

$$
\ell\left(\sum_{k=0}^{[t / \varepsilon]} \mathbb{E}_{\pi}\left[\Phi_{k+1}^{\varepsilon} \varphi-\Phi_{k}^{\varepsilon} \varphi \mid \mathcal{F}_{k}\right]\right)
$$

is relatively compact for any $\ell \in \mathbb{B}_{0}^{*}$.

Lemma 7.4. The family $\ell\left(M_{[t / \varepsilon]}^{\varepsilon} \varphi\right)$ is relatively compact for any $\ell \in \mathbb{B}_{0}^{*}$ and any $\varphi \in \mathbb{B}_{0}$.

Proof. It is worth noting that the martingale $M_{[t / \varepsilon]}^{\varepsilon}$ can be expressed in terms of martingale differences:

$$
M_{[t / \varepsilon]}^{\varepsilon}=\sum_{k=0}^{[t / \varepsilon]-1} \mathbb{E}_{\pi}\left[\Phi_{k+1}^{\varepsilon} \varphi-\mathbb{E}_{\pi}\left[\Phi_{k+1}^{\varepsilon} \varphi \mid \mathcal{F}_{k}\right]\right] .
$$

Then, using the equality

$$
\mathbb{E}_{\pi}\left[\Phi_{k+1}^{\varepsilon} \varphi \mid \mathcal{F}_{k}\right]=\mathbb{E}_{\pi}\left[D^{\varepsilon}\left(z_{k+1}\right) \Phi_{k}^{\varepsilon} \varphi \mid \mathcal{F}_{k}\right]
$$


we obtain

$$
\begin{aligned}
M_{[(t+\eta) / \varepsilon]}^{\varepsilon} \varphi-M_{[t / \varepsilon]}^{\varepsilon} \varphi & =\sum_{k=[t / \varepsilon]+1}^{[(t+\eta) / \varepsilon]}\left[D^{\varepsilon}\left(z_{k+1}\right) \Phi_{k}^{\varepsilon} \varphi-\mathbb{E}_{\pi}\left[D^{\varepsilon}\left(z_{k+1}\right) \Phi_{k}^{\varepsilon} \varphi \mid \mathcal{F}_{k}\right]\right] \\
& =\sum_{k=[t / \varepsilon]+1}^{[(t+\eta) / \varepsilon]}\left[D^{\varepsilon}\left(z_{k+1}\right) \Phi_{k}^{\varepsilon} \varphi-P^{\sharp} D^{\varepsilon}\left(z_{k+1}\right) \Phi_{k}^{\varepsilon} \varphi\right] \\
& =\sum_{k=[t / \varepsilon]+1}^{[(t+\eta) / \varepsilon]}\left[D^{\varepsilon}\left(z_{k+1}\right)-P^{\sharp} D^{\varepsilon}\left(z_{k+1}\right)\right] \Phi_{k}^{\varepsilon} \varphi
\end{aligned}
$$

for any $\eta>0$. Now, from the above, we obtain

$$
\begin{aligned}
\mathbb{E}_{\pi} \mid \ell( & \left.M_{[(t+\eta) / \varepsilon]}^{\varepsilon} \varphi-M_{[t / \varepsilon]}^{\varepsilon} \varphi\right) \mid \\
\leq & \left(\left[\frac{t+\eta}{\varepsilon}\right]-\left[\begin{array}{c}
t \\
\varepsilon
\end{array}\right]\right) \varepsilon \\
& \times \mathbb{E}_{\pi}\left[\left\|D_{1}\left(z_{k+1}\right) \varphi\right\|+\left\|D_{0}^{\varepsilon}\left(z_{k+1}\right) \varphi\right\|+\left\|P^{\sharp} D_{1}(\cdot) \varphi\right\|+\left\|P^{\sharp} D_{0}^{\varepsilon}(\cdot) \varphi\right\|\right] \\
\leq & 2 \eta\left(\left\|P^{\sharp} D_{1}(\cdot) \varphi\right\|+\left\|P^{\sharp} D_{0}^{\varepsilon}(\cdot) \varphi\right\|\right) \\
\rightarrow & 0 \text { as } \eta \rightarrow 0,
\end{aligned}
$$

which completes the proof.

Proof of Theorem 3.1. From Lemmas 7.1-7.4 and representation (7.1), it follows that the family $\Phi_{[t / \varepsilon]}^{\varepsilon} \varphi$ satisfies the compact containment condition, and that the family $\ell\left(\Phi_{[t / \varepsilon]}^{\varepsilon} \varphi\right)$ is relatively compact for any $\ell \in \mathbb{B}_{0}^{*}$ and any $\varphi \in \mathbb{B}_{0}$. Hence, the relative compactness of the family $\Phi_{[t / \varepsilon]}^{\varepsilon} \varphi$ is established.

Let $\mathbb{L}^{\mathcal{E}}(x), x \in E$, be a family of perturbed operators defined on $\mathbb{B}$ by

$$
\mathbb{L}^{\varepsilon}(x):=\varepsilon^{-1} Q^{\sharp}+P^{\sharp} D_{1}(x)+P^{\sharp} D_{0}^{\varepsilon}(x) .
$$

Then the process

$$
M_{t}^{\varepsilon}=\Phi_{[t / \varepsilon]}^{\varepsilon}-I-\varepsilon \sum_{\ell=0}^{[t / \varepsilon]-1} \mathbb{L}^{\varepsilon} \Phi_{\ell}^{\varepsilon}
$$

is an $\mathcal{F}_{t}^{\varepsilon}$-martingale.

The singular perturbation problem for the nonnegligible part of the compensating operator $\mathbb{L}^{\varepsilon}$, denoted by

$$
\mathbb{L}_{0}^{\varepsilon}(x):=\varepsilon^{-1} Q^{\sharp}+P^{\sharp} D_{1}(x),
$$

given by

$$
\mathbb{L}_{0}^{\varepsilon} \varphi^{\varepsilon}=\mathbb{L} \varphi+\varepsilon \theta^{\varepsilon},
$$

on the test functions $\varphi^{\varepsilon}(u, x)=\varphi(u)+\varepsilon \varphi_{1}(u, x)$, has the solution (see [18, Proposition 5.1]) $\varphi \in \mathcal{N}\left(Q^{\sharp}\right), \varphi_{1}=R_{0} \tilde{D}_{1} \varphi$, with $\tilde{D}_{1}(x)=P^{\sharp} D_{1}(x)-\hat{D}_{1}, \hat{D}_{1}=\int_{E} \pi(\mathrm{d} x) D_{1}(x)$, and $\theta^{\varepsilon}(x)=$ $P^{\sharp} D_{1}(x) R_{0} \tilde{D}_{1}(x) \varphi$.

The limit operator is then given by

$$
\mathbb{L} \Pi=\Pi D_{1}(\cdot) \Pi,
$$


from which we get the contracted limit operator

$$
\hat{\mathbb{L}}=\hat{D}_{1}
$$

It is worth noting that martingale $M_{t}^{\varepsilon}$ has the asymptotic representation

$$
M_{t}^{\varepsilon}=\Phi_{[t / \varepsilon]}^{\varepsilon}-I-\varepsilon \sum_{\ell=0}^{[t / \varepsilon]-1} \hat{\mathbb{L}} \Phi_{\ell}^{\varepsilon}+O_{\varphi}(\varepsilon),
$$

where $\left\|O_{\varphi}(\varepsilon)\right\| \rightarrow 0$ as $\varepsilon \rightarrow 0$. The families $l\left(M_{[t / \varepsilon]}\right)$ and $l\left(\sum_{\ell=0}^{[t / \varepsilon]-1}\left[P^{\sharp} D^{\varepsilon}(\cdot)-I\right] \Phi_{\ell}^{\varepsilon}\right)$ are weakly compact for all $l \in \mathbb{B}_{0}^{*}$ in a dense subset $\mathbb{B}_{0}^{*} \subset \mathbb{B}$. This means that the family $l\left(\Phi_{[t / \varepsilon]}^{\varepsilon}\right)$ is also weakly compact. In this way, the sum $\varepsilon \sum_{\ell=0}^{[t / \varepsilon]-1} \hat{\mathbb{L}} \Phi_{\ell}^{\varepsilon} \varphi$ converges as $\varepsilon \rightarrow 0$ to the integral $\int_{0}^{t} \hat{\mathbb{L}} \bar{\Phi}(s) \varphi \mathrm{d} s$. The quadratic variation of the martingale $l\left(M_{t}^{\varepsilon} \varphi\right)$ tends to 0 when $\varepsilon \rightarrow 0$; hence, $M_{t}^{\varepsilon} \varphi \rightarrow 0$ when $\varepsilon \rightarrow 0$ for any $f \in \mathbb{B}_{0}$ and any $l \in \mathbb{B}_{0}^{*}$. Passing to the limit in (7.3), when $\varepsilon \rightarrow 0$, we obtain $\Phi_{[t / \varepsilon]}^{\varepsilon} \varphi \rightarrow \bar{\Phi}(t) \varphi$ as $\varepsilon \rightarrow 0$, where $\bar{\Phi}(t)$ is defined in (3.1).

In the average scheme, the limit $M_{t}^{0}$ for the martingale $M_{t}^{\varepsilon}$ has a quadratic variation equal to 0 . In fact, the quadratic variation is

$$
\left\langle\ell\left(M_{[t / \varepsilon]}^{\varepsilon}\right)\right\rangle=\sum_{k=0}^{[t / \varepsilon]} \mathbb{E}_{\pi}\left[\ell^{2}\left(M_{k+1}^{\varepsilon} \varphi^{\varepsilon}-M_{k}^{\varepsilon} \varphi^{\varepsilon}\right) \mid \mathcal{F}_{k}\right],
$$

where $\varphi^{\varepsilon}(x)=\varphi(x)+\varepsilon \varphi_{1}(x)$. Hence,

$$
\ell\left(M_{k+1}^{\varepsilon} \varphi^{\varepsilon}-M_{k}^{\varepsilon} \varphi^{\varepsilon}\right)=\ell\left(\left(M_{k+1}^{\varepsilon}-M_{k}^{\varepsilon}\right) \varphi\right)+\varepsilon \ell\left(\left(M_{k+1}^{\varepsilon}-M_{k}^{\varepsilon}\right) \varphi_{1}\right)
$$

and

$$
M_{k+1}^{\varepsilon}-M_{k}^{\varepsilon}=\Phi_{k+1}^{\varepsilon}-\Phi_{k}^{\varepsilon}-\mathbb{E}_{\pi}\left[\Phi_{k+1}^{\varepsilon}-\Phi_{k}^{\varepsilon} \mid \mathcal{F}_{k}\right]
$$

Therefore,

$$
\begin{aligned}
\ell\left(M_{k+1}^{\varepsilon} \varphi^{\varepsilon}-M_{k}^{\varepsilon} \varphi^{\varepsilon}\right)= & \ell\left(\left(D\left(z_{k+1}\right)^{\varepsilon}-I\right) \Phi_{k}^{\varepsilon} \varphi\right)-\mathbb{E}_{\pi}\left[\left(D^{\varepsilon}\left(z_{k+1}\right)-I\right) \varphi \mid \mathcal{F}_{k}\right] \\
& +\varepsilon \ell\left(\left(D^{\varepsilon}\left(z_{k+1}\right)-I\right) \varphi_{1}\right)-\mathbb{E}_{\pi}\left[\left(D\left(z_{k+1}\right)^{\varepsilon}-I\right) \varphi_{1} \mid \mathcal{F}_{k}\right] \\
= & \varepsilon \ell\left(\left(D_{1}\left(z_{k+1}\right)+D_{0}^{\varepsilon}\left(z_{k+1}\right)\right) \varphi\right)-\varepsilon \mathbb{E}_{\pi}\left[\left(D_{1}\left(z_{k+1}\right)+D_{0}^{\varepsilon}\left(z_{k+1}\right)\right) \varphi \mid \mathcal{F}_{k}\right] \\
& +\varepsilon^{2} \ell\left(\left(D_{1}\left(z_{k+1}\right)+D_{0}^{\varepsilon}\left(z_{k+1}\right)\right) \varphi_{1}\right) \\
& -\varepsilon^{2} \mathbb{E}_{\pi}\left[\left(D_{1}\left(z_{k+1}\right)+D_{0}^{\varepsilon}\left(z_{k+1}\right)\right) \varphi_{1} \mid \mathcal{F}_{k}\right] .
\end{aligned}
$$

From (7.4) and (7.5) and the boundedness of the operators in (7.5) with respect to $\mathbb{E}_{\pi}$, it follows that $\left\langle\ell\left(M_{[t / \varepsilon]}^{\varepsilon}\right)\right\rangle$ goes to 0 when $\varepsilon \rightarrow 0$.

The limit martingale $M_{t}^{0}$ equals 0 . So, the limit equation for $M_{t}^{\varepsilon}$ has the form (3.1). As long as the solution of the martingale problem for the operator $\hat{\mathbb{L}}$ is unique, then it follows that the solution to (3.1) is unique as well [12, Chapter 4], [40, Chapter 7]. It is worth noting that the operator $\hat{\mathbb{L}}$ is a first-order operator $\left(\hat{D}_{1}\right.$; see (7.2)). Finally, since the operator $\hat{\mathbb{L}}$ generates a semigroup, we have $\bar{\Phi}(t) \varphi=\exp [\hat{\mathbb{L}} t] \varphi$, which is unique. 


\subsection{Proof of Theorem 3.2}

We can prove the relative compactness of the family $\Phi_{\left[t / \varepsilon^{2}\right]}^{\varepsilon}$ by following the same steps as above. However, in the case of the diffusion approximation, the limit continuous martingale $M_{0}(t)$ for the martingale $M_{t}^{\varepsilon}$ has quadratic variation that is not 0 . That is,

$$
M_{0}(t) \varphi=\Phi_{0}(t) \varphi-\varphi-\int_{0}^{t} \hat{\mathbb{L}} \Phi_{0}(s) \mathrm{d} s
$$

and $\left\langle\ell\left(M_{0}\right)\right\rangle \neq 0$ for $\ell \in \mathbb{B}_{0}^{*}$.

Furthermore, the operator $\hat{\mathbb{L}}$ defined in Theorem 3.2 is a second-order operator since it contains the operator $\hat{D}_{2}$ and $\Pi D_{1} R_{0} P^{\sharp} D_{1} \Pi$ (compare with the first-order operator $\hat{\mathbb{L}}$ in (7.2)).

Let $\mathbb{L}^{\varepsilon}(x), x \in E$, be a family of perturbed operators defined on $\mathbb{B}$ by

$$
\mathbb{L}^{\varepsilon}(x):=\varepsilon^{-2} Q^{\sharp}+\varepsilon^{-1} P^{\sharp} D_{1}(x)+P^{\sharp} D_{2}(x)+P^{\sharp} D_{0}^{\varepsilon}(x) .
$$

Then the process

$$
M_{t}^{\varepsilon}=\Phi_{\left[t / \varepsilon^{\varepsilon}\right]}^{\varepsilon}-I-\varepsilon^{2} \sum_{k=0}^{\left[t / \varepsilon^{2}\right]-1} \mathbb{L}^{\varepsilon} \Phi_{k}^{\varepsilon}
$$

is an $\mathcal{F}_{t}^{\varepsilon}$-martingale with mean value 0 .

For the nonnegligible part of the compensating operator $\mathbb{L}^{\varepsilon}$, denoted by

$$
\mathbb{L}_{0}^{\varepsilon}(x):=\varepsilon^{-2} Q^{\sharp}+\varepsilon^{-1} P^{\sharp} D_{1}(x)+P^{\sharp} D_{2}(x),
$$

consider the singular perturbation problem

$$
\mathbb{L}_{0}^{\varepsilon} \varphi^{\varepsilon}=\mathbb{L} \varphi+\varepsilon \theta^{\varepsilon}(x),
$$

where $\varphi^{\varepsilon}(u, x)=\varphi(u)+\varepsilon \varphi_{1}(u, x)+\varepsilon^{2} \varphi_{2}(u, x)$. The solution to this problem is given by the vectors (see [18, Proposition 5.2])

$$
\varphi_{1}=R_{0} P^{\sharp} D_{1}(x) \varphi, \quad \varphi_{2}=R_{0} \tilde{A} \varphi,
$$

with $\tilde{A}(x):=A(x)-\hat{A}$, and the negligible term

$$
\theta^{\varepsilon}(x)=\left[P^{\sharp} D_{1}(x)+\varepsilon P^{\sharp} D_{2}(x)\right] \varphi_{2}+P^{\sharp} D_{2}(x) \varphi_{1} .
$$

Of course, $\varphi \in \mathcal{N}\left(Q^{\sharp}\right)$.

Now the limit operator $\mathbb{L}$ is given by

$$
\mathbb{L}=P^{\sharp} D_{2}(\cdot)+P^{\sharp} D_{1}(\cdot) R_{0} P^{\sharp} D_{1}(\cdot),
$$

from which the contracted operator on the null space $\mathcal{N}\left(Q^{\sharp}\right)$ is

$$
\hat{\mathbb{L}}=\hat{D}_{2} \Pi+\Pi D_{1}(x) R_{0} P^{\sharp} D_{1}(x) \Pi .
$$

Using the balance condition (3.2), we get the limit operator.

We note that, from conditions (A5)-(A6) and (D1)-(D2), it follows that the DTSMRE $\Phi_{\left[t / \varepsilon^{2}\right]}^{\varepsilon} \varphi$ is a contractive operator in $H$ and, hence, $\left\|\Phi_{\left[t / \varepsilon^{2}\right]}^{\varepsilon} \varphi\right\|_{H}$ is a supermartingale for any $\varphi \in H$, where $\|\cdot\|_{H}$ is a norm in the Hilbert space $H$ (see [19] and [23]). Using Doob's 
inequality for the supermartingale $\left\|\Phi_{\left[t / \varepsilon^{2}\right]}^{\varepsilon}\right\|_{H}$, we obtain

$$
\mathbb{P}\left(\Phi_{\left[t / \varepsilon^{2}\right]}^{\varepsilon} \in K_{\Delta}^{1}\right) \geq 1-\Delta
$$

where $K_{\Delta}^{1}$ is a compact set in $\mathbb{B}$ and $\Delta$ is any small number. Then, under conditions (A5)-(A6) and (D1)-(D2), the sequence $M_{t}^{\varepsilon}$ is tight and is weakly compact in $D_{\mathbb{B}}[0,+\infty)$ with limit points in $C_{\mathbb{B}}[0,+\infty)$.

Also, under conditions (A5)-(A6) and (D1)-(D2), the martingale $M_{t}^{\varepsilon}$ has the asymptotic representation

$$
M_{t}^{\varepsilon} \varphi=\Phi_{\left[t / \varepsilon^{2}\right]}^{\varepsilon} \varphi-\varphi-\varepsilon^{2} \sum_{k=0}^{\left[t / \varepsilon^{2}\right]-1} \hat{\mathbb{L}} \Phi_{k}^{\varepsilon} \varphi+O_{\varphi}(\varepsilon),
$$

where $\left\|O_{\varphi}(\varepsilon)\right\| \rightarrow 0$ as $\varepsilon \rightarrow 0$. The families $l\left(M_{t}^{\varepsilon} \varphi\right)$ and $l\left(\varepsilon^{2} \sum_{k=0}^{\left[t / \varepsilon^{\varepsilon}\right]-1} \hat{\mathbb{L}} \Phi_{k}^{\varepsilon} \varphi\right)$ are weakly compact for all $l \in \mathbb{B}^{*}$ and $\varphi \in \mathbb{B}_{0}$. This means that $\Phi_{\left[t / \varepsilon^{2}\right]}^{\varepsilon}$ is also weakly compact and has a limit. If we set $\Phi_{0}(t)$ to be this limit then the sum $\varepsilon^{2} \sum_{k=0}^{\left[t / \varepsilon^{\varepsilon}\right]-1} \hat{\mathbb{L}} \Phi_{k}^{\varepsilon} \varphi$ converges to the integral $\int_{0}^{t} \hat{\mathbb{L}} \Phi_{0}(s) \varphi \mathrm{d} s$. Let $M_{0}(t)$ be a limit martingale for $M_{t}^{\varepsilon}$ when $\varepsilon \rightarrow 0$. Then, from the previous steps and (7.6), we see that

$$
M_{0}(t) \varphi=\Phi_{0}(t) \varphi-\varphi-\int_{0}^{t} \hat{\mathbb{L}} \Phi_{0}(s) \varphi \mathrm{d} s .
$$

As long as the martingale $M_{t}^{\varepsilon}$ has mean value 0 , the martingale $M_{0}(t)$ also has mean value 0 . Taking the mean value of both sides of (7.7) yields

$$
0=\mathbb{E} \Phi_{0}(t) \varphi-\varphi-\int_{0}^{t} \hat{\mathbb{L}} \mathbb{E} \Phi_{0}(t) \varphi \mathrm{d} s
$$

Solving this, we obtain

$$
\mathbb{E} \Phi_{0}(t) \varphi=\exp [\hat{\mathbb{L}} t] \varphi
$$

This means that the operator $\hat{\mathbb{L}}$ generates the semigroup $U(t):=\mathbb{E} \Phi_{0}(t) \varphi=\exp [\hat{\mathbb{L}} t] \varphi$. The uniqueness of the limit evolution $\Phi_{0}(t)$ in the diffusion approximation scheme follows from the uniqueness of the solution to the martingale problem for $\Phi_{0}(t)$ (uniqueness of the limit process under weak compactness). As long as the solution of the martingale problem for the operator $\hat{\mathbb{L}}$ is unique, then it follows that the solution of (7.8) is unique as well [12], [40].

\subsection{Proof of Theorem 3.3}

We note that $W_{t}^{\varepsilon}$ in (3.3) has the form

$$
W_{t}^{\varepsilon}=\varepsilon^{-1 / 2}\left\{\sum_{k=1}^{[t / \varepsilon]}\left[D^{\varepsilon}\left(z_{k-1}\right)-I\right] \Phi_{k}^{\varepsilon}-\int_{0}^{t} \bar{D}_{1} \bar{\Phi}(s) \mathrm{d} s\right\} .
$$

Since $\Pi\left(D_{1}-\hat{D}_{1}\right)=0$, the diffusion approximation algorithm (see Section 3.2) may be applied to the right-hand side of (7.9) using the operators $D_{2}=0$ and $\left(D_{1}(z)-\bar{D}_{1}\right)$ instead of $D_{1}(z)$. We note that the sequence $W_{t}^{\varepsilon}$ is weakly compact, completing the proof (see Sections 7.1-7.2). 


\subsection{Proof of Proposition 5.1}

The problem is to estimate

$$
\left\|\mathbb{E}_{\pi}\left[\Phi_{[t / \varepsilon]}^{\varepsilon} \varphi^{\varepsilon}\right]-\bar{\Phi}(t) \varphi\right\|
$$

for any $\varphi \in \mathbb{B}_{0}$, where $\varphi^{\varepsilon}(x)=\varphi(x)+\varepsilon \varphi_{1}(x)$.

We note that

$$
\left(P^{\sharp}-I\right) \varphi_{1}(x)=-\left(\hat{D}_{1}-P^{\sharp} D_{1}(x)\right) \varphi .
$$

As long as $\Pi\left(\hat{D}_{1}-P^{\sharp} D_{1}(x)\right) \varphi=0, \varphi \in \mathbb{B}_{0}$, the solution to (7.10) is in the domain $\mathcal{R}\left(P^{\sharp}-I\right)$, $\varphi_{1}(x)=R_{0} \tilde{D}_{1} \varphi$. Then

$$
\mathbb{E}_{\pi}\left\|\varphi_{1}(x)\right\| \leq 2\left\|R_{0}\right\| \int_{E} \pi(\mathrm{d} z)\left\|P^{\sharp} D_{1}(z) \varphi\right\|=: 2 C_{1}\left(\varphi,\left\|R_{0}\right\|\right),
$$

where $R_{0}$ is the potential operator of $Q^{\sharp}:=P^{\sharp}-I$. From here we obtain

$$
\mathbb{E}_{\pi}\left\|\left(\Phi_{[t / \varepsilon]}^{\varepsilon}-I\right) \varphi_{1}\right\| \leq 4 C_{1}\left(\varphi,\left\|R_{0}\right\|\right),
$$

since the $\Phi_{k}^{\varepsilon}$ are contractive operators.

We also note that

$$
\left\|\mathbb{E}_{\pi}\left[\varepsilon \sum_{k=0}^{[t / \varepsilon]} \hat{\mathbb{L}} \Phi_{k}^{\varepsilon} \varphi-\int_{0}^{t} \hat{\mathbb{L}} \bar{\Phi}(s) \varphi \mathrm{d} s\right]\right\| \leq \varepsilon C_{2}(t, \varphi),
$$

where

$$
C_{2}(t, \varphi):=4 T \int_{E} \pi(\mathrm{d} z)\left\|P^{\sharp} D_{1}(z) \varphi\right\|, \quad t \in[0, T] .
$$

This follows from a standard argument about the convergence of Riemann sums in the Bochner integral (see Lemma 4.14 of [19, p. 161]).

We have

$$
\left\|\mathbb{E}_{\pi}\left[\Phi_{[t / \varepsilon]}^{\varepsilon} \varphi^{\varepsilon}\right]-\bar{\Phi}(t) \varphi\right\| \leq \| \mathbb{E}_{\pi}\left[\Phi_{[t / \varepsilon]}^{\varepsilon} \varphi-\bar{\Phi}(t) \varphi \|+\varepsilon C_{1}\left(\varphi,\left\|R_{0}\right\|\right),\right.
$$

where we have used $\varphi^{\varepsilon}=\varphi+\varepsilon \varphi_{1}$. Note that $\bar{\Phi}(t)$ satisfies the equation

$$
\bar{\Phi}(t) \varphi-\varphi-\int_{0}^{t} \hat{\mathbb{L}} \bar{\Phi}(s) \varphi \mathrm{d} s=0 .
$$

Let us introduce the martingale

$$
M_{[t / \varepsilon]+1}^{\varepsilon} \varphi^{\varepsilon}:=\Phi_{[t / \varepsilon]}^{\varepsilon} \varphi^{\varepsilon}-\varphi^{\varepsilon}-\sum_{k=0}^{[t / \varepsilon]} \mathbb{E}_{\pi}\left[\Phi_{k+1}^{\varepsilon} \varphi^{\varepsilon}-\Phi_{k}^{\varepsilon} \varphi^{\varepsilon} \mid \mathcal{F}_{k}\right] .
$$

This is a zero-mean value martingale; hence,

$$
\mathbb{E}_{\pi} M_{[t / \varepsilon]}^{\varepsilon} \varphi^{\varepsilon}=0
$$


which follows directly from (7.14). It also follows from (7.14) that

$$
\begin{aligned}
M_{[t / \varepsilon]}^{\varepsilon} \varphi^{\varepsilon}= & \Phi_{[t / \varepsilon]}^{\varepsilon} \varphi-\varphi+\varepsilon\left[\Phi_{[t / \varepsilon]}-I\right] \varphi_{1}-\varepsilon \sum_{k=0}^{[t / \varepsilon]} \hat{\mathbb{L}} \Phi_{k}^{\varepsilon} \varphi \\
& -\varepsilon^{2} \sum_{k=0}^{[t / \varepsilon]}\left[P^{\sharp} D_{1}(\cdot) \Phi_{k}^{\varepsilon} \varphi_{1}+o_{\varphi}(1)\right],
\end{aligned}
$$

where $o_{\varphi}(1) \rightarrow 0$ as $\varepsilon \rightarrow 0$ for any $\varphi \in \mathbb{B}_{0}$.

Now, from (3.1) and expressions (7.15)-(7.16), we obtain

$$
\begin{aligned}
\mathbb{E}_{\pi}\left[\Phi_{[t / \varepsilon]}^{\varepsilon} \varphi-\bar{\Phi}(t) \varphi\right]= & \varepsilon \mathbb{E}_{\pi}\left[\Phi_{[t / \varepsilon]^{\varepsilon}}-I\right] \varphi_{1}+\mathbb{E}_{\pi}\left[\varepsilon \sum_{k=0}^{[t / \varepsilon]} \hat{\mathbb{L}} \Phi_{k}^{\varepsilon} \varphi-\int_{0}^{t} \hat{\mathbb{L}} \bar{\Phi}(s) \varphi \mathrm{d} s\right] \\
& +\varepsilon^{2} \mathbb{E}_{\pi}\left[\sum_{k=0}^{[t / \varepsilon]-1} R_{k}\left(\varphi_{1}\right)\right]
\end{aligned}
$$

where $R_{k}\left(\varphi_{1}\right):=P^{\sharp} D_{1}(\cdot) \Phi_{k}^{\varepsilon} \varphi_{1}+o_{\varphi}(1)$. Let us estimate $\left\|R_{k}\left(\varphi_{1}\right)\right\|$ in (7.17). We have

$$
\left\|R_{k}\left(\varphi_{1}\right)\right\| \leq \sup _{g \in K_{\Delta}}\left(\left\|P^{\sharp} D_{1}(z) g\right\|+\left\|o_{g}(1)\right\|\right)=: C_{3}\left(z, g, K_{\Delta}\right),
$$

where $K_{\Delta}$ is a compact set, $\Delta>0$, because $\Phi_{k}^{\varepsilon} \varphi_{1}$ satisfies the compactness condition for any $\varepsilon>0$ and any $k$. In this way, from (7.17) we obtain

$$
\left\|\mathbb{E}_{\pi}\left[\sum_{k=0}^{[t / \varepsilon]-1} R_{k}\left(\varphi_{1}\right)\right]\right\| \leq T \int_{E} \pi(\mathrm{d} z) C_{3}\left(z, g, K_{\Delta}\right), \quad t \in[0, T] .
$$

Finally, from inequalities (7.11)-(7.13) and (7.18)-(7.19), we obtain the desired rate of convergence of the DTSMRE in the averaging scheme, i.e.

$$
\left\|\mathbb{E}_{\pi}\left[\Phi_{[t / \varepsilon]}^{\varepsilon} \varphi^{\varepsilon}\right]-\bar{\Phi}(t) \varphi\right\| \leq \varepsilon A\left(T, \varphi,\left\|R_{0}\right\|,\left\|D_{1}\right\|\right),
$$

where the constant

$$
A\left(T, \varphi,\left\|R_{0}\right\|,\left\|D_{1}\right\|\right):=5 C_{1}\left(\varphi,\left\|R_{0}\right\|\right)+C_{2}(T, \varphi)+T \int_{E} \pi(\mathrm{d} z) C_{3}\left(z, g, K_{\Delta}\right)
$$

and $C_{3}\left(z, g, K_{\Delta}\right)$ is defined in (7.18). This completes the proof.

Remark 7.1. In a similar way, we can obtain the rate of convergence results in the diffusion approximation (see Propositions 5.2-5.3).

\subsection{Proof of Theorem 6.1}

Suppose that there is an optimal control, say $\bar{u}$, which is Markov. Let $\Delta>0$, and let $\alpha$ be any value in $\mathcal{U}$. Define $\tilde{u}_{s}$ to be the control that uses the feedback $\bar{u}$ for $k \geq \Delta$ and uses the control identically equal to $\alpha$ for $k<\Delta$. Define the CDTSMRE $\tilde{\Phi}_{k}^{\tilde{u}}$ to be the random evolution which corresponds to control $\hat{u}$. 
Let $\tilde{\tau}$ denote the time that the target set is reached under this composite control. Also, let $\Phi_{k}^{u}$ and $\hat{c}$ denote the solution and escape time under the optimal control $\bar{u}$. By definition we have

$$
C_{0}(\varphi, z, k)=\mathbb{E}_{z, k}^{\bar{u}}\left[\sum_{i=k}^{\tau} K\left(\Phi_{i}^{u} \varphi, z_{i}, u_{i}, i\right)+b\left(\Phi_{\tau}^{u} \varphi, z_{\tau}, \tau\right)\right],
$$

where $k \in \mathbb{N}$. The optimality of $C_{0}(\varphi, z, k)$ implies that

$$
\begin{aligned}
C_{0}(\varphi, z, k) \leq & \mathbb{E}_{z, k}^{\tilde{u}}\left[\sum_{i=k}^{\tau} K\left(\Phi_{i}^{u} \varphi, z_{i}, u_{i}, i\right)+b\left(\Phi_{\tau}^{u} \varphi, z_{\tau}, \tau\right)\right] \\
= & \mathbb{E}_{z, k}^{\tilde{u}}\left[\sum_{i=k}^{\tau \wedge \Delta} K\left(\Phi_{i}^{u} \varphi, z_{i}, u_{i}, i\right)+b\left(\Phi_{\tau}^{u} \varphi, z_{\tau}, \tau\right) \mathbf{1}_{\{\tau<\Delta\}}\right] \\
& +\mathbb{E}_{z, k}^{\tilde{u}}\left[\sum_{i=k}^{\tau} K\left(\Phi_{i}^{u} \varphi, z_{i}, u_{i}, i\right)+b\left(\Phi_{\tau}^{u} \varphi, z_{\tau}, \tau\right) \mathbf{1}_{\{\tau \geq \Delta\}}\right] .
\end{aligned}
$$

By the very definition of $\tilde{u}$, the optimality of $\bar{u}$, inequality (7.20) may be written as

$$
\begin{aligned}
C_{0}(\varphi, z, k) \leq \mathbb{E}_{z, k}^{\tilde{u}}\left[\sum_{i=k}^{\tau \wedge \Delta} K\left(\Phi_{i}^{u} \varphi, z_{i}, u_{i},\right)+b\left(\Phi_{\tau}^{u} \varphi, z_{\tau}, \tau\right) \mathbf{1}_{\{\tau<\Delta\}}\right. \\
\left.+C_{0}\left(\Phi_{\Delta}^{u} \varphi, z_{\Delta}, u_{\Delta}, \Delta\right) \mathbf{1}_{\{\tau \geq \Delta\}}\right]
\end{aligned}
$$

Hence,

$$
\begin{aligned}
& \frac{1}{\Delta} \mathbb{E}_{z, k}^{\tilde{u}}\left[C_{0}\left(\Phi_{\Delta}^{u} \varphi, z_{\Delta}, u_{\Delta}, k+\Delta\right)-C_{0}(\varphi, z, k)+\sum_{i=k}^{\tau+\Delta} K\left(\Phi_{i}^{u} \varphi, z_{i}, u_{i}, i\right)\right] \\
& \geq \frac{1}{\Delta} \mathbb{E}_{z, t}^{\tilde{u}} g(\tau, \Delta, \tilde{u}) \mathbf{1}_{\{\tau<\Delta\}},
\end{aligned}
$$

where

$$
g(\tau, \Delta, \tilde{u}):=C_{0}\left(\Phi_{\Delta}^{u} \varphi, z_{\Delta}, \Delta\right)-\sum_{\tau \wedge \Delta}^{\Delta} K\left(\Phi_{i}^{\bar{u}} \varphi, z_{i}, u_{i}, i\right)-b\left(\Phi_{\tau}^{\bar{u}} \varphi, z_{\tau}, \tau\right)
$$

is bounded uniformly by $\Delta$.

If we assume that $\mathbb{P}_{z, k}^{\tilde{u}}(\tau<\Delta) / \Delta \rightarrow 0$ as $\Delta \rightarrow 0$ then the right-hand side of (7.21) tends to 0 as $\Delta \rightarrow 0$. Taking this limit yields, for any $\alpha \in \mathcal{U}$,

$$
L^{\alpha} C_{0}(\varphi, z, k)+K(\varphi, z, u, k) \geq 0, \quad \varphi \in G^{0},
$$

where the operator $L^{\alpha}$ is defined in (6.1).

If we suppose in the calculation above that $\alpha$ is replaced by $\bar{u}$ on $[0, \Delta]$, then the analogue of (7.21) holds with the inequality replaced by an equality. Then we obtain the equation

$$
L^{\bar{u}} C_{0}(\varphi, z, k)+K(\varphi, z, u, k)=0, \quad \varphi \in G^{0} .
$$


From here, it follows that

$$
\begin{gathered}
\inf _{\alpha \in \mathcal{U}}\left[L^{\alpha} C_{0}(\varphi, z, k)+K(\varphi, z, \alpha, k)\right]=0, \quad \varphi \in G^{0}, \\
C_{0}(\varphi, z, k)=b(\varphi, z, k), \quad \varphi \in \partial G .
\end{gathered}
$$

In this way, we obtain the HJB equation for the CDTSMRE.

Let us prove the converse result. Suppose that we have a bounded function $C_{0}(\varphi, z, k)$ on $C_{b}\left(G^{0}\right)$ and control $\bar{u}$ such that (7.22) holds. Let us show that $C_{0}(\varphi, z, k)$ is indeed the optimal cost and $\bar{u}$ is an optimal control. Let $\tilde{u}$ be any adapted control with $\Phi_{t}^{\tilde{u}}$ a CDTSMRE.

By the minimization in (7.22),

$$
L^{\bar{u}} C_{0}(\varphi, z, k)+K(\varphi, z, \bar{u}, k)=0, \quad \varphi \in G^{0},
$$

and

$$
L^{\tilde{u}} C_{0}(\varphi, z, k)+K(\varphi, z, \tilde{u}, k) \geq 0, \quad \varphi \in G^{0} .
$$

Let $\tau$ and $\tilde{\tau}$ be the escape times under the controls $u$ and $\tilde{u}$. Then, from the definitions of the operators $L^{\bar{u}}$ and $L^{\tilde{u}}$, we can write (which follows from Dynkin's formula for a CDTSMRE)

$$
\begin{aligned}
-\mathbb{E}_{z, k}^{\bar{u}} C_{0}\left(\Phi_{l \wedge \tau}^{\bar{u}} \varphi, z_{l \wedge \tau}, l \wedge \tau\right)+C_{0}(\varphi, z, k) & =-\mathbb{E}_{z, k}^{\bar{u}} \sum_{i=k}^{l \wedge \tau} L^{\bar{u}} C_{0}\left(\Phi_{i}^{\bar{u}} \varphi, z_{i}, i\right) \\
& =\sum_{i=k}^{l \wedge \tau} K\left(\Phi_{i}^{\bar{u}} \varphi, z_{i}, \bar{u}_{i}, i\right), \quad l>k
\end{aligned}
$$

and

$$
\begin{aligned}
-\mathbb{E}_{z, k}^{\tilde{u}} C_{0}\left(\Phi_{l \wedge \tau}^{\tilde{u}} \varphi, z_{l \wedge \tilde{\tau}}, l \wedge \tilde{\tau}\right)+C_{0}(\varphi, z, k) & =-\mathbb{E}_{z, t}^{\tilde{u}} \sum_{i=k}^{l \wedge \tilde{\tau}} L^{\tilde{u}} C_{0}\left(\Phi_{i}^{\tilde{u}} \varphi, z_{i}, i\right) \\
& \leq \sum_{i=k}^{l \wedge \tilde{\tau}} K\left(\Phi_{i}^{\tilde{u}} \varphi, z_{i}, \tilde{u}_{i}, i\right), \quad l>k .
\end{aligned}
$$

We note that

$$
\mathbb{E}_{z, k}^{\bar{u}} C_{0}\left(\Phi_{l \wedge \tau}^{\bar{u}} \varphi, z_{l \wedge \tau}, l \wedge \tau\right) \rightarrow \mathbb{E}_{z, k}^{\bar{u}} b\left(\Phi_{\tau}^{\bar{u}} \varphi, z_{\tau}, \tau\right) \quad \text { as } l \rightarrow \infty
$$

and

$$
\mathbb{E}_{z, k}^{\tilde{u}} C_{0}\left(\Phi_{l \wedge \tilde{\tau}}^{\tilde{u}} \varphi, z_{l \wedge \tilde{\tau}}, l \wedge \tilde{\tau}\right) \rightarrow \mathbb{E}_{z, k}^{\tilde{u}} b\left(\Phi_{\tau}^{\tilde{u}} \varphi, z_{\tilde{\tau}}, \tilde{\tau}\right) \quad \text { as } l \rightarrow \infty .
$$

From (7.23) and (7.25), and (7.24) and (7.26), we finally obtain

$$
C(\varphi, z, \bar{u}, k)=\mathbb{E}_{z, k}^{\bar{u}}\left[\sum_{i=k}^{\tau} K\left(\Phi_{i}^{\bar{u}} \varphi, z_{i}, \bar{u}_{i}, i\right)+b\left(\Phi_{\tau}^{\bar{u}} \varphi, z_{\tau}, \tau\right)\right]=C_{0}(\varphi, z, k)
$$

and

$$
C_{0}(\varphi, z, k) \leq \mathbb{E}_{z, k}^{\tilde{u}}\left[\sum_{i=k}^{\tilde{\tau}} K\left(\Phi_{i}^{\tilde{u}} \varphi, z_{i}, \tilde{u}_{i}, i\right)+b\left(\Phi_{\tilde{\tau}}^{\bar{u}} \varphi, z_{\tau}, \tau\right)\right]=C(\varphi, z, \tilde{u}, k),
$$

respectively. In this way, from (7.27) and (7.28), we show that the minimizing control $\bar{u}$ is optimal, completing the proof. 


\section{Concluding remarks and future work}

In this paper we introduced discrete-time semi-Markov random evolutions (DTSMREs) and studied asymptotic properties, namely, averaging, diffusion approximation, and diffusion approximation with equilibrium by the martingale weak convergence method. The controlled DTSMREs were also introduced and Hamilton-Jacobi-Bellman equations derived for them. Applications were given to additive functionals and geometric Markov renewal processes. The rates of convergence in the limit theorems for DTSMREs, additive functionals, and geometric Markov renewal chains were also presented.

In this paper we considered fixed initial points for the processes. In fact, the same results hold when we replace this fixed condition $u$ by $y^{\varepsilon}(0), \varepsilon>0$, and the conditions $y^{\varepsilon}(0) \stackrel{\mathbb{P}}{\rightarrow} y(0)$ and $\sup _{\varepsilon>0} \mathbb{E}\left|y^{\varepsilon}(0)\right|<+\infty$. It is worth noting that the vector case $\mathbb{R}^{d}$ can be written, starting from the presented results, directly.

Future work will concentrate on the study of the stability of DTSMREs and applications of the obtained results to reliability theory, DNA analysis, and other discrete-time applied models. We will also study merging problems for DTSMREs, in particular, merging in averaging and diffusion approximation schemes, as well as general stopping problems for DTSMREs.

\section{Acknowledgements}

We are indebted to an anonymous referee for useful comments that improved the presentation of this paper. The research of the second author was partially supported by the NSERC. The second author also thanks the Laboratory of Applied Mathematics at the Universite de Technologie de Compiègne for their hospitality during his two-week visit in June/July 2011. The first author thanks the Department of Mathematics and Statistics at the University of Calgary for their hospitality during his one-week visit in May 2012.

\section{References}

[1] AASE, K. K. (1988). Contingent claims valuation when the security price is a combination of an Itô process and a random point process. Stoch. Process. Appl. 28, 185-220.

[2] Adams, R. (1979). Sobolev Spaces. Academic Press, New York.

[3] Altman, E. And Shwartz, A. (1991). Markov decision problems and state-action frequencies. SIAM J. Control Optimization 29, 786-809.

[4] Anisimov, V. V. (2008). Switching Processes in Queueing Models. John Wiley, Hoboken, NJ.

[5] Barbu, V. S. and Limnios, N. (2008). Semi-Markov Chains and Hidden Semi-Markov Models. Toward Applications (Lecture Notes Statist. 191). Springer, New York.

[6] Bertsekas, D. P. And Shreve, S. E. (1996). Stochastic Optimal Control. Athena Scientific, Belmont, MA.

[7] Beutler, F. J. AND Ross, K. W. (1985). Optimal policies for controlled Markov chains with a constraint. J. Math. Anal. Appl. 112, 236-252.

[8] Borkar, V. S. (2003). Dynamic programming for ergodic control with partial observations. Stoch. Process. Appl. 103, 293-310.

[9] Boussement, M. And Limnios, N. (2004). Markov decision processes with asymptotic average failure rate constraint. Commun. Statist. Theory Meth. 33, 1689-1714.

[10] Chiquet, J., Limnios, N. And Eid, M. (2009). Piecewise deterministic Markov processes applied to fatigue crack growth modelling. J. Statist. Planning Infer. 139, 1657-1667.

[11] Cox, J. C., Ross, S. A. And Rubinstein, M. (1979). Option pricing: a simplified approach. J. Financial Econom. 7, 229-263.

[12] Ethier, S. N. And Kurtz, T. G. (1986). Markov Processes. John Wiley, New York.

[13] Fleming, W. H. and Rishel, R. W. (1975). Deterministic and Stochastic Optimal Control. Springer, Berlin.

[14] Hersh, R. (1974). Random evolutions: a survey of results and problems. Rocky Mountain J. Math. 4, $443-477$.

[15] JACOD, J. ANd Shiryaev, A. N. (1987). Limit Theorems for Stochastic Processes. Springer, Berlin.

[16] JAŚKIEWICZ, A. AND NowAK, A. S. (2007). Average optimality for semi-Markov control processes. Morfismos 11, 15-36. 
[17] Keepler, M. (1998). Random evolutions processes induced by discrete time Markov chains. Portugal. Math. 55, 391-400.

[18] Koroliuk, V. S. And Limnios, N. (2005). Stochastic Systems in Merging Phase Space. World Scientific, Hackensack, NJ.

[19] Korolyuk, V. S. And SwishchuK, A. (1995). Evolution of Systems in Random Media. CRC Press, Boca Raton, FL.

[20] Krylov, N. ANd Bogolyubov, N. (1947). Introduction to Non-Linear Mechanics. Princeton University Press, Princeton.

[21] Kushner, H. (1971). Introduction to Stochastic Control. Holt, Rinehart and Winston, New York.

[22] Ledoux, M. and Talangrand, M. (1991). Probability in Banach Spaces. Springer, Berlin.

[23] Limnios, N. (2011). Discrete-time semi-Markov random evolutions-average and diffusion approximation of difference equations and additive functionals. Commun. Statist. Theory Meth. 40, 3396-3406.

[24] Limnios, N. and Oprişan, G. (2001). Semi-Markov Processes and Reliability. Birkhäuser, Boston, MA.

[25] Maxwell, M. and Woodroofe, M. (2000). Central limit theorems for additive functionals of Markov chains. Ann. Prob. 28, 713-724.

[26] Meyn, S. P. And Tweedie, R. L. (1993). Markov Chains and Stochastic Stability. Springer, London.

[27] Nummelin, E. (1984). General Irreducible Markov Chains and Nonnegative Operators. Cambridge University Press.

[28] PInsky, M. A. (1991). Lectures on Random Evolution. World Scientific, River Edge, NJ.

[29] Pyke, R. (1961). Markov renewal processes: definitions and preliminary properties. Ann. Math. Statist. 32, 1231-1242.

[30] Pyke, R. (1961). Markov renewal processes with finitely many states. Ann. Math. Statist. 32, 1243-1259.

[31] Pyke, R. and Schaufele, R. (1964). Limit theorems for Markov renewal processes. Ann. Math. Statist. 35, 1746-1764.

[32] Revuz, D. (1975). Markov Chains. North-Holland, Amsterdam.

[33] Rudin, W. (1991). Functional Analysis. McGraw-Hill, New York.

[34] Shurenkov, V. M. (1984). On the theory of Markov renewal. Theory Prob. Appl. 19, 247-265.

[35] Silvestrov, D. S. (1991). The invariance principle for accumulation processes with semi-Markov switchings in a scheme of arrays. Theory Prob. Appl. 36, 519-535.

[36] Silvestrov, D. S. (2004). Limit Theorems for Randomly Stopped Stochastic Processes. Springer, London.

[37] Skorokнod, A. V. (1989). Asymptotic Methods in the Theory of Stochastic Differential Equations. American Mathematical Society, Providence, RI.

[38] Skorokhod, A. V., Hoppensteadt, F. C. And Salehi, H. (2002). Random Perturbation Methods with Applications in Science and Engineering. Springer, New York.

[39] Sobolev, S. L. (1991). Some Applications of Functional Analysis in Mathematical Physics. American Mathematical Society, Providence, RI.

[40] Stroock, D. W. and Varadhan, S. R. S. (1979). Multidimensional Diffusion Processes. Springer, Berlin.

[41] Sviridenko, M. N. (1989). Martingale approach to limit theorems for semi-Markov processes. Theory Prob. Appl. 34, 540-545.

[42] SwishchuK, A. V. (1995). Random Evolutions and Their Applications. Kluwer, Dordrecht.

[43] Swishchuk, A. And Islam, M. S. (2010). The geometric Markov renewal processes with application to finance. Stoch. Anal. Appl. 29, 684-705.

[44] Swishchuk, A. And Islam, M. S. (2010). Diffusion approximations of the geometric Markov renewal processes and option price formulas. Internat. J. Stoch. Anal. 2010, 347105, 21 pp.

[45] SwishchuK, A. V. And Islam, M. S. (2013). Normal deviation and Poisson approximation of GMRP. To appear in Commun. Statist. Theory Meth.

[46] SwishchuK, A. V. AND Limnios, N. (2011). Optimal stopping of GMRP and pricing of European and American options. In Proc. 15th Internat. Congress on Insurance: Mathematics and Economics (Trieste, Italy, June 2011).

[47] Swishchuk, A. And Wu, J. (2003). Evolution of Biological Systems in Random Media: Limit Theorems and Stability. Kluwer, Dordrecht.

[48] Vega-Amaya, O. And Luque-VÁsquez, F. (2000). Sample-path average cost optimality for semi-Markov control processes on Borel spaces: unbounded costs and mean holding times. Appl. Math. 27, 343-367.

[49] Yin, G. G. AND Zhang, Q. (2005). Discrete-Time Markov Chains. Springer, New York. 